\title{
Unconditional Reflexive Polytopes
}

\section{Florian Kohl ${ }^{1} \cdot$ McCabe Olsen $^{2} \cdot$ Raman Sanyal $^{3}$}

Received: 2 June 2019 / Revised: 6 February 2020 / Accepted: 16 March 2020 / Published online: 16 April 2020 () The Author(s) 2020

\begin{abstract}
A convex body is unconditional if it is symmetric with respect to reflections in all coordinate hyperplanes. We investigate unconditional lattice polytopes with respect to geometric, combinatorial, and algebraic properties. In particular, we characterize unconditional reflexive polytopes in terms of perfect graphs. As a prime example, we study the signed Birkhoff polytope. Moreover, we derive constructions for Gale-dual pairs of polytopes and we explicitly describe Gröbner bases for unconditional reflexive polytopes coming from partially ordered sets.
\end{abstract}

Keywords Unconditional polytopes - Reflexive polytopes · Unimodular triangulations $\cdot$ Perfect graphs $\cdot$ Gale-dual pairs $\cdot$ Signed Birkhoff polytopes

Mathematics Subject Classification 52B20 - 52B12 - 52B15 - 05C17

Dedicated to the memory of Branko Grünbaum

Editor in Charge: Kenneth Clarkson

The first author was supported by the Academy of Finland Project Numbers 288318 and 13324921. The third author was supported by the DFG-Collaborative Research Center, TRR 109 "Discretization in Geometry and Dynamics".

Florian Kohl

florian.kohl@aalto.fi

McCabe Olsen

olsen.149@osu.edu

Raman Sanyal

sanyal@math.uni-frankfurt.de

1 Department of Mathematics and Systems Analysis, Aalto University, Espoo, Finland

2 Department of Mathematics, The Ohio State University, Columbus, OH 43210, USA

3 Institut für Mathematik, Goethe-Universität, Frankfurt am Main, Germany 


\section{Introduction}

A $d$-dimensional convex lattice polytope $P \subset \mathbb{R}^{d}$ is called reflexive if its polar dual $P^{*}$ is again a lattice polytope. Reflexive polytopes were introduced by Batyrev [6] in the context of mirror symmetry as a reflexive polytope and its dual give rise to a mirrordual pair of Calabi-Yau manifolds; cf. [22]. As thus, the results of Batyrev, and the subsequent connection with string theory, have stimulated interest in the classification of reflexive polytopes both among mathematical and theoretical physics communities. As a consequence of a well-known result of Lagarias and Ziegler [47], there are only finitely many reflexive polytopes in each dimension, up to unimodular equivalence. In two dimensions, it is a straightforward exercise to verify that there are precisely 16 reflexive polygons, as depicted in Fig. 1. While still finite, there are significantly more reflexive polytopes in higher dimensions. Kreuzer and Skarke $[45,46]$ have completely classified reflexive polytopes in dimensions 3 and 4, noting that there are exactly 4319 reflexive polytopes in dimension 3 and 473,800,776 reflexive polytopes in dimension 4. The number of reflexive polytopes in dimension 5 is not known.

In recent years, there has been significant progress in characterizing reflexive polytopes in known classes of polytopes coming from combinatorics or optimization; see, for example, $[7,21,42,54,73]$. The purpose of this paper is to study a class of reflexive polytopes motivated by convex geometry and relate it to combinatorics. A convex body $K \subset \mathbb{R}^{d}$ is unconditional if $\boldsymbol{p} \in K$ if and only if $\sigma \boldsymbol{p}:=\left(\sigma_{1} p_{1}, \sigma_{2} p_{2}, \ldots, \sigma_{d} p_{d}\right) \in K$ for all $\sigma \in\{ \pm 1\}^{d}$. Unconditional convex bodies, for example, arise as unit balls in the theory of Banach spaces with a 1-unconditional basis. They constitute a restricted yet surprisingly interesting class of convex bodies for which a number of claims
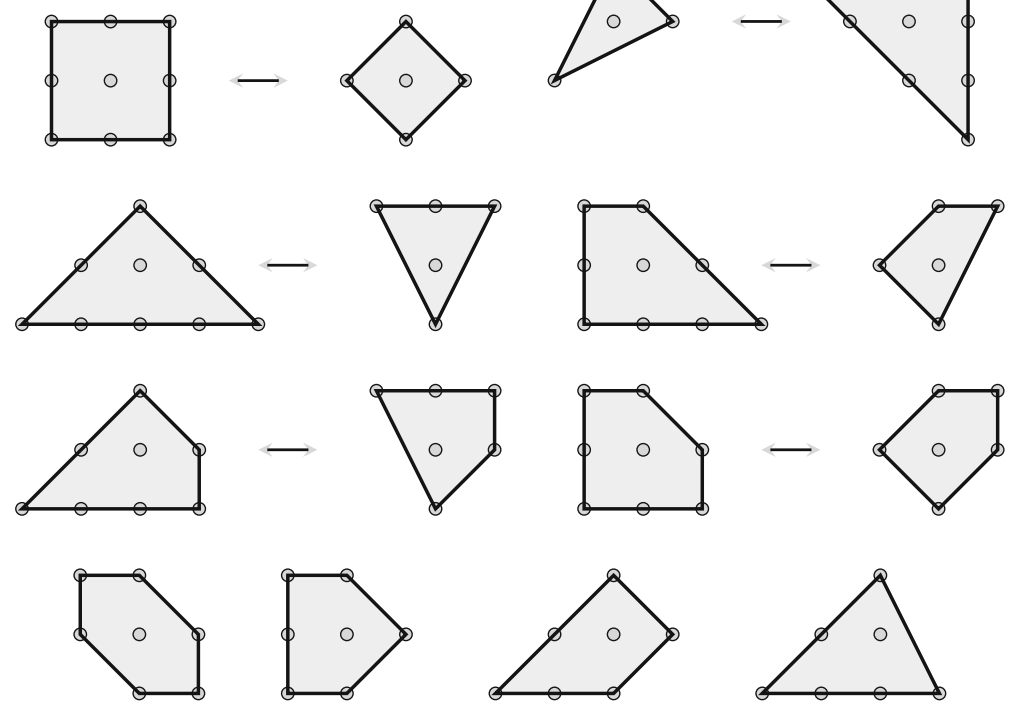

Fig. 1 All 16 reflexive 2-dimensional polytopes. This is [43, Fig. 1.5] 
have been verified; cf. [14]. For example, we mention that the Mahler conjecture is known to hold for unconditional convex bodies; see Sect. 3. In this paper, we investigate unconditional lattice polytopes and their relation to anti-blocking polytopes from combinatorial optimization. In particular, we completely characterize unconditional reflexive polytopes.

The structure of this paper is as follows. In Sect. 2, we briefly review notions and results from discrete geometry and Ehrhart theory. In Sect. 3, we introduce and study unconditional and, more generally, locally anti-blocking polytopes. The main result is Theorem 3.2 which relates regular, unimodular, and flag triangulations to the associated anti-blocking polytopes. In Sect. 4, we associate an unconditional lattice polytope $\cup P_{G}$ to every finite graph $G$. We show in Theorems 4.6 and 4.9 that an unconditional polytope $P$ is reflexive if and only if $P=\cup P_{G}$ for some unique perfect graph $G$. This also implies that unconditional reflexive polytopes have regular, unimodular triangulations. Section 5 is devoted to a particular family of unconditional reflexive polytopes and is of independent interest: We show that the type-B Birkhoff polytope or signed Birkhoff polytope $\mathcal{B B}(n)$, that is, the convex hull of signed permutation matrices, is an unconditional reflexive polytope. We compute normalized volumes and $h^{*}$-vectors of $\mathcal{B B}(n)$ and its dual $\mathcal{C}(n):=\mathcal{B B}(n)^{*}$ for small values of $n$. The usual Birkhoff polytope and the Gardner polytope of [32] appear as faces of $\mathcal{B B}(n)$ and $\mathcal{C}(n)$, respectively. These two polytopes form a Gale-dual pair in the sense of [32]. In Sect. 6, we give a general construction for compressed Gale-dual pairs coming from CIS graphs. In Sect. 7, we investigate unconditional polytopes associated to comparability graphs of posets. In particular, we explicitly describe a quadratic square-free Gröbner basis for the corresponding toric ideal. We close with open questions and future directions in Sect. 8.

\section{Background}

In this section, we provide a brief introduction to polytopes and Ehrhart theory. For additional background and details, we refer the reader to the excellent books $[9,75]$. A polytope in $\mathbb{R}^{d}$ is the inclusion-minimal convex set $P=\operatorname{conv}\left(\boldsymbol{v}_{1}, \ldots, \boldsymbol{v}_{n}\right)$ containing a given collection of points $\boldsymbol{v}_{1}, \ldots, \boldsymbol{v}_{n} \in \mathbb{R}^{d}$. The unique inclusion-minimal set $V \subseteq P$ such that $P=\operatorname{conv}(V)$ is called the vertex set and is denoted by $V(P)$. If $V(P) \subset \mathbb{Z}^{d}$, then $P$ is called a lattice polytope. By the Minkowski-Weyl theorem, polytopes are precisely the bounded sets of the form

$$
P=\left\{\boldsymbol{x} \in \mathbb{R}^{d}:\left\langle\boldsymbol{a}_{i}, \boldsymbol{x}\right\rangle \leq b_{i} \text { for } i=1, \ldots, m\right\}
$$

for some $\boldsymbol{a}_{1}, \ldots, \boldsymbol{a}_{m} \in \mathbb{R}^{d}$ and $b_{1}, \ldots, b_{m} \in \mathbb{R}$. If $\left\langle\boldsymbol{a}_{i}, \boldsymbol{x}\right\rangle \leq b_{i}$ is irredundant, then $F=P \cap\left\{\boldsymbol{x}:\left\langle\boldsymbol{a}_{i}, \boldsymbol{x}\right\rangle=b_{i}\right\}$ is a facet and the inequality is said to be facet-defining.

The dimension of a polytope $P$ is defined to be the dimension of its affine span. A $d$-dimensional polytope has at least $d+1$ vertices and a $d$-polytope with exactly $d+1$ many vertices is called a $d$-simplex. A $d$-simplex $\Delta=\operatorname{conv}\left\{\boldsymbol{v}_{0}, \boldsymbol{v}_{1}, \ldots, \boldsymbol{v}_{d}\right\}$ is called unimodular if $\boldsymbol{v}_{1}-\boldsymbol{v}_{0}, \boldsymbol{v}_{2}-\boldsymbol{v}_{0}, \ldots, \boldsymbol{v}_{d}-\boldsymbol{v}_{0}$ form a basis for the lattice $\mathbb{Z}^{d}$, or, equivalently, if $\operatorname{vol}(\Delta)=1 / d$ !, where vol is the Euclidean volume. For lattice 
polytopes $P \subset \mathbb{R}^{d}$, we define the normalized volume $\operatorname{Vol}(P):=d ! \operatorname{vol}(P)$. So unimodular simplices are the lattice polytopes with normalized volume 1 . We say that two lattice polytopes $P, P^{\prime} \subset \mathbb{R}^{d}$ are unimodularly equivalent if $P^{\prime}=T(P)$ for some transformation $T(\boldsymbol{x})=W \boldsymbol{x}+\boldsymbol{v}$ with $W \in \mathrm{SL}_{d}(\mathbb{Z})$ and $\boldsymbol{v} \in \mathbb{Z}^{d}$. In particular, any two unimodular simplices are unimodularly equivalent.

Given a lattice $e$-polytope $P \subset \mathbb{R}^{d}$ and $t \in \mathbb{Z}_{\geq 1}$, let $t P:=\{t \cdot \boldsymbol{x}: \boldsymbol{x} \in P\}$ be the $t^{\text {th }}$ dilate of $P$. By a famous result of Ehrhart [30, Thm. 1], the lattice-point enumeration function

$$
\operatorname{ehr}_{P}(t):=\left|t P \cap \mathbb{Z}^{d}\right|
$$

agrees with a polynomial in the variable $t$ of degree $e$ with leading coefficient $\operatorname{vol}(P)$ and is called the Ehrhart polynomial. This also implies that the formal generating function

$$
1+\sum_{t \geq 1} \operatorname{ehr}_{P}(t) z^{t}=\frac{h_{0}^{*}(P)+h_{1}^{*}(P) z+\cdots+h_{e}^{*}(P) z^{e}}{(1-z)^{e+1}}
$$

is a rational function such that the degree of the numerator is at most $e$ (see, e.g., [9, Lem. 3.9]). We call the numerator the $h^{*}$-polynomial of $P$. The vector $h^{*}(P)=$ $\left(h_{0}^{*}(P), h_{1}^{*}(P), \ldots, h_{d}^{*}(P)\right) \in \mathbb{Z}^{d+1}$, where we set $h_{i}^{*}(P):=0$ for $\operatorname{dim} P<i \leq d$, is called the $h^{*}$-vector of $P$. One should note that the Ehrhart polynomial is invariant under unimodular transformations.

Theorem 2.1 ([67,70]) Let $P \subseteq Q \subset \mathbb{R}^{d}$ be lattice polytopes. Then

$$
0 \leq h_{i}^{*}(P) \leq h_{i}^{*}(Q)
$$

for all $i=0, \ldots, d$.

The $h^{*}$-vector encodes significant information about the underlying polytope. This is nicely illustrated in the case of reflexive polytopes. For a $d$-polytope $P \subset \mathbb{R}^{d}$ with 0 in the interior, we define the (polar) dual polytope

$$
P^{*}:=\left\{\boldsymbol{y} \in \mathbb{R}^{d}:\langle\boldsymbol{y}, \boldsymbol{x}\rangle \leq 1 \text { for all } \boldsymbol{x} \in P\right\} .
$$

Definition 2.2 Let $P \subset \mathbb{R}^{d}$ be a $d$-dimensional lattice polytope that contains the origin in its interior. We say that $P$ is reflexive if $P^{*}$ is also a lattice polytope. Equivalently, $P$ is reflexive if it has a description of the form

$$
P=\left\{\boldsymbol{x} \in \mathbb{R}^{d}:\left\langle\boldsymbol{a}_{i}, \boldsymbol{x}\right\rangle \leq 1 \text { for } i=1, \ldots, m\right\},
$$

for some $\boldsymbol{a}_{1}, \ldots, \boldsymbol{a}_{m} \in \mathbb{Z}^{d}$.

Reflexivity can be completely characterized by enumerative data of the $h^{*}$-vector. 
Theorem 2.3 ([41, Thm. 2.1]) Let $P \subset \mathbb{R}^{d}$ be a d-dimensional lattice polytope with $h^{*}(P)=\left(h_{0}^{*}, \ldots, h_{d}^{*}\right)$. Then $P$ is unimodularly equivalent to a reflexive polytope if and only if $h_{k}^{*}=h_{d-k}^{*}$ for all $0 \leq k \leq\lfloor d / 2\rfloor$.

The reflexivity property is also deeply related to commutative algebra. A polytope $P \subset \mathbb{R}^{d}$ is reflexive if the canonical module of the associated graded algebra $\mathbf{k}[P]$ is (up to a shift in grading) isomorphic to $\mathbf{k}[P]$ and its minimal generator has degree 1. If one allows the unique minimal generator to have arbitrary degree, one arrives at the notion of Gorenstein rings, for details we refer to [17, Sect. 6.C]. We say that $P$ is Gorenstein if there exist $c \in \mathbb{Z}_{\geq 1}$ and $\boldsymbol{q} \in \mathbb{Z}^{d}$ such that $\boldsymbol{q}+c P$ is a reflexive polytope. This is equivalent to saying that $\mathbf{k}[P]$ is Gorenstein. The dilation factor $c$ is often called the codegree. In particular, reflexive polytopes are Gorenstein of codegree 1. By combining results of Stanley [66] and De Negri-Hibi [27], we have a characterization of the Gorenstein property in terms of the $h^{*}$-vector. Namely, $P$ is Gorenstein if and only if $h_{i}^{*}=h_{d-c+1-i}^{*}$ for all $i$.

Aside from examining algebraic properties of lattice polytopes, one can also investigate discrete geometric properties. Every lattice polytope admits a subdivision into lattice simplices. Even more, one can guarantee that every lattice point contained in a polytope corresponds to a vertex of such a subdivision. However, one cannot guarantee the existence of a subdivision where all simplices are unimodular when the dimension is greater than 2. This leads us to our next definition:

Definition 2.4 Let $P$ be a $d$-dimensional lattice polytope given by $P=\operatorname{conv}(V)$ for some finite set $V \subset \mathbb{Z}^{d}$. A subdivision of $P$ with vertices in $V$ is a collection $\mathcal{S}=$ $\left\{P_{1}, \ldots, P_{r}\right\}$ of $d$-dimensional polytopes with vertices in $V$ such that $P=P_{1} \cup \cdots \cup P_{r}$ and $P_{i} \cap P_{j}$ is a common face of $P_{i}$ and $P_{j}$ for all $1 \leq i<j \leq r$. If all polytopes $P_{i}$ are (unimodular) simplices, then $\mathcal{S}$ is a (unimodular) triangulation. A subdivision $\mathcal{S}^{\prime}=\left\{P_{1}^{\prime}, \ldots, P_{s}^{\prime}\right\}$ refines $\mathcal{S}$ if for every $P_{i}^{\prime} \in \mathcal{S}^{\prime}$ there is $P_{j} \in \mathcal{S}$ such that $P_{i}^{\prime} \subseteq P_{j}$.

Suppose that $P=\operatorname{conv}(V)$. Any map $\omega: V \rightarrow \mathbb{R}$ yields a piecewise-linear and convex function $\bar{\omega}: P \rightarrow \mathbb{R}$ by $\bar{\omega}(p):=\min \left\{\lambda:(p, \lambda) \in P^{\omega}\right\}$, where $P^{\omega}:=$ conv $((v, \omega(v)): v \in V)$. The domains of linearity of $\bar{\omega}$ determine a subdivision of $P$, called a regular subdivision. The function $\omega$ is referred to as heights on $P$. For more on (regular) subdivisions and details we refer to [26,37].

A particularly simple example of a regular triangulation of a (lattice) polytope $P$ is the pulling triangulation. For an arbitrary but fixed ordering of the vertices $V(P)$, let $\boldsymbol{v}_{F}$ be the first vertex in the face $F$ in the given ordering. The pulling triangulation of $P$ is then defined recursively as follows: If $P$ is a simplex, then $\mathcal{T}=\{P\}$. Otherwise, let $F_{1}, \ldots, F_{r}$ be the facets of $P$ not containing $\boldsymbol{v}_{P}$ and let $\mathcal{T}_{1}, \ldots, \mathcal{T}_{r}$ be their pulling triangulations with respect to the induced ordering. Then

$$
\mathcal{T}:=\left\{\operatorname{conv}\left(\left\{\boldsymbol{v}_{P}\right\} \cup S\right): S \in \mathcal{T}_{j}, j=1, \ldots, r\right\}
$$

is the pulling triangulation of $P$; see [10, Ch. 5.7].

For a subdivision $\mathcal{S}$, we write $V(\mathcal{S})=\bigcup_{Q \in \mathcal{S}} V(Q)$. If $\mathcal{T}$ is a triangulation, then $W \subseteq V(\mathcal{T})$ is called a non-face if $\operatorname{conv}(W)$ is not a face of any $Q \in \mathcal{T}$. The triangulation $\mathcal{T}$ is called flag if the inclusion-minimal non-faces $W$ satisfy $|W|=2$. 
A special class of polytopes which possess regular, unimodular triangulations are compressed polytopes. A polytope $P$ is compressed if every pulling triangulation is unimodular [67]. In the interest of providing a useful characterization of compressed polytopes, we must define the notion of width with respect to a facet. Let $P \subset \mathbb{R}^{d}$ be a $d$-dimensional lattice polytope and $F_{i}=P \cap\left\{\boldsymbol{x}:\left\langle\boldsymbol{a}_{i}, \boldsymbol{x}\right\rangle=b_{i}\right\}$ a facet. We assume that $\boldsymbol{a}_{i}$ is primitive, that is, its coordinates are coprime. The width of $P$ with respect to the facet $F_{i}$ is

$$
\max _{\boldsymbol{p} \in P}\left\langle\boldsymbol{a}_{i}, \boldsymbol{p}\right\rangle-\min _{\boldsymbol{p} \in P}\left\langle\boldsymbol{a}_{i}, \boldsymbol{p}\right\rangle
$$

The maximum over all facets is called the facet width of $P$.

Theorem 2.5 ([55, Thm. 1.1], [72, Thm. 2.4]) Let $P \subset \mathbb{R}^{d}$ be a full-dimensional lattice polytope. The following are equivalent:

(i) $P$ is compressed;

(ii) $P$ has facet width one;

(iii) $P$ is unimodularly equivalent to the intersection of a unit cube with an affine space.

Definition 2.6 A lattice polytope $P \subset \mathbb{R}^{d}$ has the integer decomposition property (IDP) if for any positive integer $t$ and for all $\boldsymbol{x} \in t P \cap \mathbb{Z}^{d}$, there exist $\boldsymbol{v}_{1}, \ldots, \boldsymbol{v}_{t} \in$ $P \cap \mathbb{Z}^{d}$ such that $\boldsymbol{x}=\boldsymbol{v}_{1}+\cdots+\boldsymbol{v}_{t}$.

One should note that if $P$ has a unimodular triangulation, then $P$ has the IDP. However, there are examples of polytopes which have the IDP, yet do not even admit a unimodular cover, that is, a covering of $P$ by unimodular simplices, see $[16$, Sec. 3]. A more complete hierarchy of covering properties can be found in [37].

We say that $h^{*}(P)$ is unimodal if there exists a $k$ such that $h_{0}^{*} \leq h_{1}^{*} \leq \cdots \leq$ $h_{k}^{*} \geq \cdots \geq h_{d-1}^{*} \geq h_{d}^{*}$. Unimodality appears frequently in combinatorial settings and it often hints at a deeper underlying algebraic structure, see $[1,15,69]$. One famous instance is given by Gorenstein polytopes that admit a regular, unimodular triangulation.

Theorem 2.7 ([19, Thm. 1]) If $P$ is Gorenstein and has a regular, unimodular triangulation, then $h^{*}(P)$ is unimodal.

The following conjecture is commonly attributed to Ohsugi and Hibi [56]:

Conjecture 2.8 If $P$ is Gorenstein and has the IDP, then $h^{*}(P)$ is unimodal.

\section{Unconditional and Anti-blocking Polytopes}

For $\sigma \in\{ \pm 1\}^{d}$ and $\boldsymbol{p} \in \mathbb{R}^{d}$, let us write $\sigma \boldsymbol{p}:=\left(\sigma_{1} p_{1}, \sigma_{2} p_{2}, \ldots, \sigma_{d} p_{d}\right)$. A convex polytope $P \subset \mathbb{R}^{d}$ is called 1-unconditional or simply unconditional if $\boldsymbol{p} \in P$ implies $\sigma p \in P$ for all $\sigma \in\{ \pm 1\}^{d}$. So, unconditional polytopes are precisely the polytopes that are invariant under reflection in all coordinate hyperplanes. It is apparent that $P$ can 
be recovered from its restriction to the first orthant $\mathbb{R}_{+}^{d}:=\left\{x \in \mathbb{R}^{d}: x_{1}, \ldots, x_{d} \geq 0\right\}$, which we denote by $P_{+}:=P \cap \mathbb{R}_{+}^{d}$. The polytope $P_{+}$has the property that for any $\boldsymbol{q} \in P_{+}$and $\boldsymbol{p} \in \mathbb{R}^{d}$ with $0 \leq p_{i} \leq q_{i}$ for all $i$, it holds that $\boldsymbol{p} \in P_{+}$. Polytopes in $\mathbb{R}_{+}^{d}$ with this property are called anti-blocking polytopes. Anti-blocking polytopes were studied and named by Fulkerson $[33,34]$ in the context of combinatorial optimization, but they are also known as convex corners or down-closed polytopes; see, for example, [13].

Let us also write $\overline{\boldsymbol{p}}:=\left(\left|p_{1}\right|,\left|p_{2}\right|, \ldots,\left|p_{d}\right|\right)$. Given an anti-blocking polytope $Q \subset \mathbb{R}_{+}^{d}$ it is straightforward to verify that

$$
\cup Q:=\left\{\boldsymbol{p} \in \mathbb{R}^{d}: \overline{\boldsymbol{p}} \in Q\right\}
$$

is an unconditional convex body. Following Schrijver's treatment of anti-blocking polytopes in [64, Sec. 9.3], we recall that every full-dimensional anti-blocking polytope has an irredundant inequality description of the form

$$
Q=\left\{\boldsymbol{x} \in \mathbb{R}_{+}^{d}:\left\langle\boldsymbol{a}_{i}, \boldsymbol{x}\right\rangle \leq 1 \text { for } i=1, \ldots, m\right\}
$$

for some $\boldsymbol{a}_{1}, \ldots, \boldsymbol{a}_{m} \in \mathbb{R}_{+}^{d}$. Also, we define

$$
\left\{\boldsymbol{c}_{1}, \ldots, \boldsymbol{c}_{r}\right\}^{\downarrow}:=\mathbb{R}_{+}^{d} \cap\left(\operatorname{conv}\left(\boldsymbol{c}_{1}, \ldots, \boldsymbol{c}_{r}\right)+\left(-\mathbb{R}_{+}^{d}\right)\right),
$$

where ' + ' denotes vector sum, as the inclusion-minimal anti-blocking polytope containing the points $\boldsymbol{c}_{1}, \ldots, \boldsymbol{c}_{r} \in \mathbb{R}_{+}^{d}$. Conversely, if we define $V^{\downarrow}(Q):=\left\{\boldsymbol{v}_{1}, \ldots, \boldsymbol{v}_{r}\right\}$ to be the vertices of an anti-blocking polytope $Q$ that are maximal with respect to the componentwise order, then $Q=\left\{\boldsymbol{v}_{1}, \ldots, \boldsymbol{v}_{r}\right\}^{\downarrow}$. We record the consequences for the unconditional polytopes.

Proposition 3.1 Let $P \subset \mathbb{R}_{+}^{d}$ be an anti-blocking d-polytope given by (1). Then an irredundant inequality description of $U P$ is given by the distinct

$$
\left\langle\sigma \boldsymbol{a}_{i}, \boldsymbol{x}\right\rangle \leq 1
$$

for $i=1, \ldots, m$ and $\sigma \in\{ \pm 1\}^{d}$. Likewise, the vertices of $U P$ are $V(U P)=\{\sigma v$ : $\left.\boldsymbol{v} \in V^{\downarrow}(P), \sigma \in\{ \pm 1\}^{d}\right\}$.

Our first result relates properties of subdivisions of anti-blocking polytopes to that of the associated unconditional polytopes. The $2^{d}$ orthants in $\mathbb{R}^{d}$ are denoted by $\mathbb{R}_{\sigma}^{d}:=\sigma \mathbb{R}_{+}^{d}$ for $\sigma \in\{ \pm 1\}^{d}$.

Theorem 3.2 Let $P \subset \mathbb{R}_{+}^{d}$ be an anti-blocking polytope with triangulation $\mathcal{T}$. Then

$$
U \mathcal{T}:=\left\{\sigma S: S \in \mathcal{T}, \sigma \in\{ \pm 1\}^{d}\right\}
$$

is a triangulation of UP. Furthermore:

(i) If $\mathcal{T}$ is unimodular, then so is $U \mathcal{T}$. 
(ii) If $\mathcal{T}$ is regular, then so is $U \mathcal{T}$.

(iii) If $\mathcal{T}$ is flag, then so is $U \mathcal{T}$.

Proof It is clear that $U \mathcal{T}$ is a triangulation of $U P$ and statement (i) is obvious.

To show (iii), let us assume that $\mathcal{T}$ is flag and let $W \subseteq V(\mathrm{U} \mathcal{T})$ be an inclusionminimal non-face of $\mathrm{U} \mathcal{T}$. If $W \subset \mathbb{R}_{\sigma}^{d}$ for some $\sigma$, then $\sigma W \subseteq V(\mathcal{T})$ is an inclusionminimal non-face of $\mathcal{T}$ and hence $|W|=|\sigma W|=2$. Thus, there are $1 \leq i \leq d$ and $\boldsymbol{p}, \boldsymbol{p}^{\prime} \in W$ with $p_{i}<0<p_{i}^{\prime}$. But then $W^{\prime}=\left\{\boldsymbol{p}, \boldsymbol{p}^{\prime}\right\} \subseteq W$ is also a non-face. Since we assume $W$ to be inclusion-minimal, this proves (iii).

To show (ii), assume that $\mathcal{T}$ is regular and let $\omega: V(P) \rightarrow \mathbb{R}$ the corresponding heights. We extend $\omega$ to $V:=\bigcup_{\sigma} \sigma V(\mathcal{T})$ by setting $\omega^{\prime}(\boldsymbol{v}):=\|\boldsymbol{v}\|_{1}+\varepsilon \omega(\overline{\boldsymbol{v}})$, where $\|\boldsymbol{v}\|_{1}=\sum_{i}\left|v_{i}\right|$ and $\overline{\boldsymbol{v}}=\left(\left|v_{1}\right|, \ldots,\left|v_{d}\right|\right)$. For $\varepsilon=0$ it is easy to see that the heights induce a regular subdivision of $P$ into $\sigma P_{+}$for $\sigma \in\{ \pm 1\}^{d}$. For $\varepsilon>0$ sufficiently small, the heights $\omega^{\prime}$ then induce the triangulation $\sigma \mathcal{T}$ on $\sigma P$.

We call a polytope $P \subset \mathbb{R}^{d}$ locally anti-blocking if $(\sigma P) \cap \mathbb{R}_{+}^{d}$ is an anti-blocking polytope for every $\sigma \in\{ \pm 1\}^{d}$. In particular, every locally anti-blocking polytope comes with a canonical subdivision into polytopes $P_{\sigma}:=P \cap \mathbb{R}_{\sigma}^{d}$ for $\sigma \in\{ \pm 1\}^{d}$. Unconditional polytopes and anti-blocking polytopes are both clearly locally antiblocking. It follows from [21, Lem. 3.12] that for any two anti-blocking polytopes $P_{1}, P_{2} \subset \mathbb{R}_{+}^{d}$, the polytopes

$$
P_{1}+\left(-P_{2}\right) \quad \text { and } \quad P_{1} \vee\left(-P_{2}\right):=\operatorname{conv}\left(P_{1} \cup-P_{2}\right)
$$

are locally anti-blocking. Locally anti-blocking polytopes are studied in depth in [3]. The following is a simple, but important observation.

Lemma 3.3 Let $P \subset \mathbb{R}^{d}$ be a locally anti-blocking lattice polytope with 0 in the interior. Then $P$ is reflexive if and only if $P_{\sigma}=P \cap \mathbb{R}_{\sigma}^{d}$ is compressed for all $\sigma \in\{ \pm 1\}^{d}$.

Proof Since $P$ is a lattice polytope with 0 in the interior, we can assume that $P$ is given as

$$
P=\left\{\boldsymbol{x} \in \mathbb{R}^{d}:\left\langle\boldsymbol{a}_{i}, \boldsymbol{x}\right\rangle \leq b_{i} \text { for } i=1, \ldots, m\right\}
$$

for some $\boldsymbol{a}_{1}, \ldots, \boldsymbol{a}_{m} \in \mathbb{Z}^{d}$ primitive and $b_{1}, \ldots, b_{m} \in \mathbb{Q}_{>0}$. Note that for $\sigma \in\{ \pm 1\}^{d}$ we have that $P_{\sigma}=P \cap \mathbb{R}_{\sigma}^{d}$ is given by all $\boldsymbol{x} \in \mathbb{R}_{\sigma}^{d}$ such that

$$
\left\langle\boldsymbol{a}_{i}, \boldsymbol{x}\right\rangle \leq b_{i} \quad \text { for } i \in I_{\sigma},
$$

where $I_{\sigma} \subseteq\left\{i \in[m]: \boldsymbol{a}_{i} \in \mathbb{R}_{\sigma}^{d}\right\}$ so that the inequalities are facet-defining. For $\sigma \in\{ \pm 1\}^{d}$ and $i \in I_{\sigma}$, we observe

$$
\min _{\boldsymbol{p} \in P_{\sigma}}\left\langle\boldsymbol{a}_{i}, \boldsymbol{p}\right\rangle=0 \quad \text { and } \quad \max _{\boldsymbol{p} \in P_{\sigma}}\left\langle\boldsymbol{a}_{i}, \boldsymbol{p}\right\rangle=b_{i}
$$

The former holds because $\left\langle\boldsymbol{a}_{i}, \boldsymbol{p}\right\rangle \geq 0$ for $\boldsymbol{a}_{i}, \boldsymbol{p} \in \mathbb{R}_{\sigma}^{d}$ and the latter because $\left\langle\boldsymbol{a}_{i}, \boldsymbol{p}\right\rangle=b_{i}$ is facet-defining. Hence $P_{\sigma}$ has facet width one with respect to $a_{i}$ if and only if $b_{i}=1$. 
Since for every $i \in[m]$ there is a $\sigma \in\{ \pm 1\}^{d}$ with $i \in I_{\sigma}$, we get using Theorem 2.5 that if $P_{\sigma}$ is compressed, then $P$ has a representation as in Definition 2.2 and is therefore reflexive.

Now let $P$ be reflexive and $\sigma$ arbitrary. We only need to argue that the facets of $P_{\sigma}$ corresponding to $\left\{\boldsymbol{x}: x_{j}=0\right\}$ have facet width one. Assume that there is a vertex $\boldsymbol{v} \in P_{\sigma}$ with $\sigma_{j} v_{j}>1$ for some $j \in[d]$. Since $P_{\sigma}$ is bounded, we can find $i \in I_{\sigma}$ with $\left(a_{i}\right)_{j} \neq 0$. Since $\sigma \boldsymbol{a}_{i} \in \mathbb{Z}_{\geq 0}^{d}$, we compute $1 \geq\left\langle\boldsymbol{a}_{i}, \boldsymbol{v}\right\rangle \geq\left(a_{i}\right)_{j} v_{j}>1$, which is a contradiction. Hence all facets of $P_{\sigma}$ have facet width one and, by Theorem 2.5, $P_{\sigma}$ is compressed.

Theorem 3.4 If $P$ is a reflexive and locally anti-blocking polytope, then $P$ has a regular and unimodular triangulation. In particular, $h^{*}(P)$ is unimodal.

Proof By definition of locally anti-blocking polytopes, $P$ is subdivided into the polytopes $P_{\sigma}$ for $\sigma \in\{ \pm 1\}^{d}$. As is easily seen, this is a regular subdivision with respect to the height function $\omega(\boldsymbol{v}):=\|\boldsymbol{v}\|_{1}$.

Let $U:=P \cap \mathbb{Z}^{d}$. By Lemma 3.3, the polytopes $P_{\sigma}$ are compressed and thus, using Theorem 2.5, $U \cap \mathbb{R}_{\sigma}^{d}$ is the vertex set of $P_{\sigma}$. Fix a order on $U$. Since $P_{\sigma}$ is compressed, the pulling triangulation $\mathcal{T}_{\sigma}$ of $P_{\sigma}$ is unimodular and we only need to argue that $\mathcal{T}=\bigcup_{\sigma} \mathcal{T}_{\sigma}$ is a regular triangulation of $P$. Let $\sigma, \sigma^{\prime} \in\{ \pm 1\}^{d}$ and let $S \in \mathcal{T}_{\sigma}$ and $S^{\prime} \in \mathcal{T}_{\sigma^{\prime}}$ be two simplices. Then $S \cap S^{\prime}$ is contained in $F=P_{\sigma} \cap P_{\sigma^{\prime}}$, which is a face of $P_{\sigma}$ as well as of $P_{\sigma^{\prime}}$. By the construction of a pulling triangulation, we see that both $S \cap F$ and $S^{\prime} \cap F$ are simplices of the pulling triangulation of $F$ and hence $S \cap S^{\prime}=(S \cap F) \cap\left(S^{\prime} \cap F\right)$ is a face of both $S$ and $S^{\prime}$. The same argument as in the proof of Theorem 3.2 then shows that $\mathcal{T}$ is a regular triangulation. The unimodality of $h^{*}(P)$ now follows from Theorem 2.7.

Remark 3.5 The techniques of this section can be extended to the following class of polytopes. We say that a polytope $P \subset \mathbb{R}^{d}$ has the orthant-lattice property (OLP) if the restriction $P_{\sigma}=P \cap \mathbb{R}_{\sigma}^{d}$ is a (possibly empty) lattice polytope for all $\sigma \in\{ \pm 1\}^{d}$. If $P$ is reflexive, then $P_{\sigma}$ is full-dimensional for every $\sigma$. Now, if every $P_{\sigma}$ has a unimodular cover, then so does $P$ and hence is IDP. Let $P_{\sigma}=\left\{\boldsymbol{x} \in \mathbb{R}_{\sigma}^{d}: A^{\sigma} \boldsymbol{x} \leq b^{\sigma}\right\}$. Then some conditions that imply the existence of a unimodular cover include:

(i) $P_{\sigma}$ is compressed;

(ii) $A^{\sigma}$ is a totally unimodular matrix;

(iii) $A^{\sigma}$ consists of rows which are $B_{d}$ roots;

(iv) $P_{\sigma}$ is the product of unimodular simplices;

(v) there exists a projection $\pi: \mathbb{R}^{d} \rightarrow \mathbb{R}^{d-1}$ such that $\pi\left(P_{\sigma}\right)$ has a regular, unimodular triangulation $\mathcal{T}$ such that the pullback subdivision $\pi^{*}(\mathcal{T})$ is lattice.

We refer to [37] for background and details. An example of such a polytope is

$$
P=\operatorname{conv}\left[\begin{array}{ccccccccc}
1 & 0 & 0 & 1 & 0 & 1 & -1 & 0 & 0 \\
0 & 1 & 0 & 1 & 1 & 1 & 0 & -1 & 0 \\
0 & 0 & 1 & 0 & 1 & 1 & 0 & 0 & -1
\end{array}\right] \subset \mathbb{R}^{3}
$$


This is a reflexive OLP polytope. The restriction to $\mathbb{R}_{+}^{3}$ is

$$
P_{+}=\operatorname{conv}\left[\begin{array}{lllllll}
1 & 0 & 0 & 1 & 0 & 1 & 0 \\
0 & 1 & 0 & 1 & 1 & 1 & 0 \\
0 & 0 & 1 & 0 & 1 & 1 & 0
\end{array}\right] \subset \mathbb{R}^{3}
$$

which is not an anti-blocking polytope.

The Mahler conjecture in convex geometry states that every centrally-symmetric convex body $K \subset \mathbb{R}^{d}$ satisfies

$$
\operatorname{vol}(K) \cdot \operatorname{vol}\left(K^{*}\right) \geq \operatorname{vol}\left(C_{d}\right) \cdot \operatorname{vol}\left(C_{d}^{*}\right)
$$

where $C_{d}=[-1,1]^{d}$ is the $d$-cube. The Mahler conjecture has been verified only in small dimensions and for special classes of convex bodies. In particular, SaintRaymond [61] proved the following beautiful inequality, where $A(P)$ refers to the anti-blocking dual of $P$, see Sect. 4. The characterization of the equality case is independently due to Meyer [51] and Reisner [60].

Theorem 3.6 (Saint-Raymond) Let $P \subset \mathbb{R}_{+}^{d}$ be an anti-blocking polytope. Then

$$
\operatorname{vol}(P) \cdot \operatorname{vol}(A(P)) \geq \frac{1}{d !}
$$

with equality if and only if $P$ or $A(P)$ is the cube $[0,1]^{d}$.

This inequality directly implies the Mahler conjecture for unconditional reflexive polytopes, which we record for the normalized volume.

Corollary 3.7 Let $P \subset \mathbb{R}^{d}$ be an unconditional reflexive polytope. Then

$$
\operatorname{Vol}(P) \cdot \operatorname{Vol}\left(P^{*}\right) \geq 4^{d} d !
$$

with equality if and only if $P$ or $P^{*}$ is the cube $[-1,1]^{d}$.

\section{Unconditional Reflexive Polytopes and Perfect Graphs}

For $A \subseteq[d]$, let $\mathbf{1}_{A} \in\{0,1\}^{d}$ be its characteristic vector. If $\Gamma \subseteq 2^{[d]}$ is a simplicial complex, that is, a nonempty set system closed under taking subsets, then

$$
P=\operatorname{conv}\left(\mathbf{1}_{\sigma}: \sigma \in \Gamma\right)
$$

is an anti-blocking 0/1-polytope and every anti-blocking polytope with vertices in $\{0,1\}^{d}$ arises that way (cf. [34, Thm. 2.3]). 
A prominent class of anti-blocking 0/1-polytopes arises from graphs. Given a graph $G=([d], E)$ with $E \subseteq\left(\begin{array}{c}{[d]} \\ 2\end{array}\right)$, we say that $S \subseteq[d]$ is a stable set (or independent set) of $G$ if $u v \notin E$ for any $u, v \in S$. The stable set polytope of $G$ is

$$
P_{G}:=\operatorname{conv}\left\{\mathbf{1}_{S}: S \subseteq[d] \text { a stable set of } G\right\}
$$

Since the stable sets of a graph form a simplicial complex, $P_{G}$ is an anti-blocking polytope. Stable set polytopes played an important role in the proof of the weak perfect graph conjecture [48]. A clique is a set $C \subseteq[d]$ such that every two vertices in $C$ are joined by an edge. The clique number $\omega(G)$ is the largest size of a clique in $G$. A graph is perfect if $\omega(H)=\chi(H)$ for all induced subgraphs $H \subseteq G$, where $\chi(H)$ is the chromatic number of $H$.

Lovász [48] gave the following geometric characterization of perfect graphs; see also [36, Thm. 9.2.4]. For a set $C \subseteq[d]$ and $\boldsymbol{x} \in \mathbb{R}^{d}$, we write $\boldsymbol{x}(C):=\left\langle\mathbf{1}_{C}, \boldsymbol{x}\right\rangle=$ $\sum_{i \in C} x_{i}$

Theorem 4.1 A graph $G=([d], E)$ is perfect if and only if

$$
P_{G}=\left\{\boldsymbol{x} \in \mathbb{R}_{+}^{d}: \boldsymbol{x}(C) \leq 1 \text { for all cliques } C \subseteq[d]\right\}
$$

For an anti-blocking polytope $P \subset \mathbb{R}_{+}^{d}$ define the anti-blocking dual

$$
A(P):=\left\{\boldsymbol{y} \in \mathbb{R}_{+}^{d}:\langle\boldsymbol{y}, \boldsymbol{x}\rangle \leq 1 \text { for all } \boldsymbol{x} \in P\right\} .
$$

The polar dual $(\mathrm{U} P)^{*}$ is again unconditional and it follows that $(\mathrm{U} P)^{*}=\mathrm{U} A(P)$.

Theorem 4.2 ([64, Thm. 9.4]) Let $P \subset \mathbb{R}_{+}^{d}$ be a full-dimensional anti-blocking polytope with

$$
P=\left\{\boldsymbol{c}_{1}, \ldots, \boldsymbol{c}_{r}\right\}^{\downarrow}=\left\{\boldsymbol{x} \in \mathbb{R}_{+}^{n}:\left\langle\boldsymbol{d}_{i}, \boldsymbol{x}\right\rangle \leq 1 \text { for all } i=1, \ldots, s\right\}
$$

for some $c_{1}, \ldots, \boldsymbol{c}_{r}, \boldsymbol{d}_{1}, \ldots, \boldsymbol{d}_{s} \in \mathbb{R}_{+}^{d}$. Then

$$
A(P)=\left\{\boldsymbol{d}_{1}, \ldots, \boldsymbol{d}_{s}\right\}^{\downarrow}=\left\{\boldsymbol{x} \in \mathbb{R}_{+}^{d}:\left\langle\boldsymbol{c}_{i}, \boldsymbol{x}\right\rangle \leq 1 \text { for all } i=1, \ldots, r\right\} .
$$

In particular, $A(A(P))=P$.

Theorems 4.1 and 4.2 then imply for a perfect graph $G$ that

$$
A\left(P_{G}\right)=\left\{\mathbf{1}_{C}: C \text { a clique in } G\right\}^{\downarrow}=P_{\bar{G}},
$$

where $\bar{G}=\left([d],\left(\begin{array}{c}{[d]} \\ 2\end{array}\right) \backslash E\right)$ is the complement graph.

Corollary 4.3 (Weak perfect graph theorem) A graph $G$ is perfect if and only if $\bar{G}$ is perfect.

We note that, in particular, if $G$ is perfect, then $P_{G}$ is compressed. 
Proposition 4.4 ([21, Prop. 3.10]) Let $P \subset \mathbb{R}_{+}^{d}$ be an anti-blocking polytope. Then $P$ is compressed if and only if $P=P_{G}$ for some perfect graph $G$.

Let us remark that Theorem 4.1 also allows us to characterize the Gorenstein stable set polytopes. For comparability graphs of posets (see Sect. 7) this was noted by Hibi [40]. A graph $G$ is called well-covered if every inclusion-maximal stable set has the same size. It is called co-well-covered if $\bar{G}$ is well-covered.

Proposition 4.5 ([56, Theorem 2.1b]) Let $P_{G}$ be the stable set polytope of a perfect graph $G=([d], E)$. Then $P_{G}$ is Gorenstein if and only if $G$ is co-well-covered.

Proof By definition, $P_{G}$ is Gorenstein if there are $c \in \mathbb{Z}_{>0}$ and $\boldsymbol{q} \in \mathbb{Z}^{d}$ such that $\boldsymbol{q}+c P_{G}$ is reflexive. Using Theorem 4 .1, we see that $\boldsymbol{q}+c P_{G}$ is given by all points $\boldsymbol{x} \in \mathbb{R}^{d}$ such that

$$
\begin{aligned}
& -x_{i} \leq-q_{i} \text { for } i=1, \ldots, d \text { and } \\
& \quad \boldsymbol{x}(C) \leq c+\boldsymbol{q}(C) \text { for all maximal cliques } C \subseteq[d] .
\end{aligned}
$$

These inequalities are facet-defining, as can be easily seen. Using the representation given in Definition 2.2, we note that $\boldsymbol{q}+c P_{G}$ is reflexive if and only if $q_{i}=-1$ for all $i=1, \ldots, d$ and $c+\boldsymbol{q}(C)=c-|C|=1$ for all maximal cliques $C$. This happens if and only if all maximal cliques have the same size.

Combining Lemma 3.3 with Proposition 4.4 yields the following characterization of reflexive locally anti-blocking polytopes.

Theorem 4.6 Let $P \subset \mathbb{R}^{d}$ be a locally anti-blocking lattice polytope with 0 in its interior. Then $P$ is reflexive if and only if for every $\sigma \in\{ \pm 1\}^{d}$ there is a perfect graph $G_{\sigma}$ such that $P_{\sigma}=\sigma P_{G_{\sigma}}$. In particular, $P$ is an unconditional reflexive polytope if and only if $P=U P_{G}$ for some perfect graph $G$.

The following corollary to Theorem 4.6 was noted in [21, Thm. 3.4]. The second part also appears in [57, Exam. 2.3].

Corollary 4.7 If $G_{1}, G_{2}$ are perfect graphs on the vertex set $[d]$, then $P_{G_{1}}+\left(-P_{G_{2}}\right)$ and $P_{G_{1}} \vee\left(-P_{G_{2}}\right)$ are reflexive polytopes.

For $G_{1}=G_{2}=K_{d}$ the complete graph on $d$ vertices, the polytope $P_{G_{1}}+\left(-P_{G_{2}}\right)$ is the Legendre polytope studied by Hetyei et al. [29,39].

Using NORMALIZ [18] and the Kreuzer-Skarke database for reflexive polytopes $[45,46]$, we were able to verify that 72 of the 3 -dimensional reflexive polytopes and at least 407 of the 4-dimensional reflexive polytopes with at most 12 vertices are locally anti-blocking. Unfortunately, our computational resources were too limited to test most of the 4-dimensional polytopes. However, there are only 114 -dimensional unconditional reflexive polytopes (by virtue of Theorem 4.9).

If $G, G^{\prime}$ are perfect graphs, then $G \uplus G^{\prime}$ as well as its bipartite sum $G \bowtie G^{\prime}=$ $\overline{\bar{G} \uplus \overline{G^{\prime}}}$ are perfect. On the level of unconditional polytopes we note that

$$
\mathrm{U} P_{G \uplus G^{\prime}}=\mathrm{U} P_{G} \times \cup P_{G^{\prime}} \quad \text { and } \quad \mathrm{U} P_{G \bowtie G^{\prime}}=\mathrm{U} P_{G} \oplus \mathrm{U} P_{G^{\prime}},
$$


where $\oplus$ is the direct sum (or free sum) of polytopes [38]. These observations give us the class of Hanner polytopes which are important in relation to the $3^{d}$-conjecture; see [62]. A centrally symmetric polytope $H \subset \mathbb{R}^{d}$ is called a Hanner polytope if and only if $H=[-1,1]$ or $H$ is of the form $H_{1} \times H_{2}$ or $H_{1} \oplus H_{2}=\left(H_{1}^{*} \times H_{2}^{*}\right)^{*}$ for lower dimensional Hanner polytopes $H_{1}, H_{2}$. Thus, every Hanner polytope is of the form $\cup P_{G}$ for some perfect graph $G$. Hanner polytopes were obtained from split graphs in [31] using a different geometric construction.

Let us briefly note that Theorem 4.6 also yields bounds on the entries of the $h^{*}$-vector. Recall that $h_{i}^{*}\left(C_{d}\right)$ for the cube $C_{d}=[-1,1]^{d}$ is given by the type- $B$ Eulerian number $B(d, i)=\sum_{j=1}^{i}(-1)^{i-j}\left(\begin{array}{c}d \\ i-j\end{array}\right)(2 j-1)^{d-1}$, which counts signed permutations with $i$ descents (see also Sect. 5). Its polar $C_{d}^{*}$ is the crosspolytope with $h_{i}^{*}\left(C_{d}^{*}\right)=\left(\begin{array}{c}d \\ i\end{array}\right)$ for $i=0, \ldots, d$.

Corollary 4.8 Let $P \subset \mathbb{R}^{d}$ be an unconditional reflexive polytope. Then

$$
\left(\begin{array}{l}
d \\
i
\end{array}\right) \leq h_{i}^{*}(P) \leq B(d, i) .
$$

Proof It follows from Theorem 4.6 that every reflexive and unconditional $P$ satisfies $C_{d}^{*} \subseteq P \subseteq C_{d}$, where $C_{d}=[-1,1]^{d}$. By Theorem 2.1, the entries of the $h^{*}$-vector are monotone with respect to inclusion.

We close the section by showing that distinct perfect graphs yield distinct unconditional reflexive polytopes.

Theorem 4.9 Let $G, H$ be perfect graphs on vertices $[d]$. Then $U P_{G}$ is unimodularly equivalent to $U P_{H}$ if and only if $G \cong H$.

Proof Assume that $T\left(\mathrm{U} P_{G}\right)=\cup P_{H}$ for some $T(\boldsymbol{x})=W \boldsymbol{x}+\boldsymbol{t}$ with $\boldsymbol{t} \in \mathbb{Z}^{d}$ and $W \in \mathrm{SL}_{d}(\mathbb{Z})$. Since the origin is the only interior lattice point of both polytopes, we infer that $t=0$. Let $W=\left(\mathbf{w}_{1}, \ldots, \mathbf{w}_{d}\right)$. Thus, $z \in \mathbb{Z}^{d}$ is a lattice point in $\cup P_{H}$ if and only if there is a stable set $S$ and $\sigma \in\{ \pm 1\}^{S}$ such that

$$
z=\sum_{i \in S} \sigma_{i} \mathbf{w}_{i}
$$

On the one hand, this implies that $\mathbf{w}_{i}$ and $\mathbf{w}_{j}$ have disjoint supports whenever $i, j \in S$ and $i \neq j$. Indeed, if the supports of $\mathbf{w}_{i}$ and $\mathbf{w}_{j}$ are not disjoint, then $\sigma_{i} \mathbf{w}_{i}+\sigma_{j} \mathbf{w}_{j}$ has a coordinate $>1$ for some choice of $\sigma_{i}, \sigma_{j} \in\{ \pm 1\}$, which contradicts the fact that $\mathrm{U} P_{H} \subseteq[-1,1]^{d}$.

On the other hand, for any $h \in[d]$, the point $\boldsymbol{e}_{h}=\mathbf{1}_{\{h\}}$ is contained in $U P_{H}$. Hence, there is a stable set $S$ and $\sigma \in\{ \pm 1\}^{S}$ such that (3) holds for $z=\boldsymbol{e}_{h}$. Since the supports of the vectors indexed by $S$ are disjoint, this means that $S=\{i\}$ and $\boldsymbol{e}_{h}=\sigma_{i} \mathbf{w}_{i}$. We conclude that $W$ is a signed permutation matrix and $G \cong H$.

We can conclude that the number of unconditional reflexive polytopes in $\mathbb{R}^{d}$ up to unimodular equivalence is precisely the number of unlabeled perfect graphs on $d$ vertices. This number has been computed up to $d=13$ (see [44, Sect. 5] and A052431 of [65]). We show the sequence in Table 1. 
Table 1 Number $p(n)$ of unlabeled perfect graphs; OEIS sequence A052431

\begin{tabular}{llllllllllll}
\hline$n$ & 3 & 4 & 5 & 6 & 7 & 8 & 9 & 10 & 11 & 12 & 13 \\
\hline$p(n)$ & 4 & 11 & 33 & 148 & 906 & 8887 & 136756 & 3269264 & 115811998 & 5855499195 & 410580177259 \\
\hline
\end{tabular}

\section{The Type-B Birkhoff Polytope}

The Birkhoff polytope $\mathcal{B}(n)$ is defined as the convex hull of all $n \times n$ permutation matrices. Equivalently, $\mathcal{B}(n)$ is the set of all doubly stochastic matrices, that is, nonnegative matrices $M$ with row and column sums equal to 1, by work of Birkhoff [11] and, independently, von Neumann [52]. This polytope has been studied quite extensively and is known to have many properties of interest (see, e.g., [4,5,8,20,24,25,59]). Of particular interest to our purposes, it is known to be Gorenstein, to be compressed [67], and to be $h^{*}$-unimodal [4]. In this section, we will introduce a type- $B$ analogue of this polytope corresponding to signed permutation matrices and verify many similar properties already known for $\mathcal{B}(n)$.

The hyperoctahedral group is defined by $B_{n}:=(\mathbb{Z} / 2 \mathbb{Z})$ ? $\mathfrak{S}_{n}$, which is the Coxeter group of type- $B$ (or type- $C$ ). Elements of this group can be thought of as permutations from $\mathfrak{S}_{n}$ expressed in one-line notation $\sigma=\sigma_{1} \sigma_{2} \ldots \sigma_{n}$, where we also associate a $\operatorname{sign} \operatorname{sgn}\left(\sigma_{i}\right)$ to each $\sigma_{i}$. To each signed permutation $\sigma \in B_{n}$, we associate a matrix $M_{\sigma}$ defined as $\left(M_{\sigma}\right)_{i, \sigma_{i}}=\operatorname{sgn}\left(\sigma_{i}\right)$ and $\left(M_{\sigma}\right)_{i, j}=0$ otherwise. If every entry of $\sigma$ is positive, then $M_{\sigma}$ is simply a permutation matrix. This leads to the following definition:

Definition 5.1 The type-B Birkhoff polytope (or signed Birkhoff polytope) is

$$
\mathcal{B B}(n):=\operatorname{conv}\left\{M_{\sigma}: \sigma \in B_{n}\right\} \subset \mathbb{R}^{n \times n} .
$$

That is, $\mathcal{B B}(n)$ is the convex hull of all $n \times n$ signed permutation matrices.

This polytope was previously studied in [50], though the emphasis was not on Ehrhart-theoretic questions. Since all points in the definition of $\mathcal{B B}(n)$ lie on a sphere, it follows that they are all vertices.

Proposition 5.2 For every $\sigma \in B_{n}, M_{\sigma}$ is a vertex of $\mathcal{B B}(n)$.

It is clear that $\mathcal{B B}(n)$ is an unconditional lattice polytope in $\mathbb{R}^{d \times d}$ and we study it by restriction to the positive orthant.

Definition 5.3 For $n \geq 1$, we define the positive type-B Birkhoff polytope, $\mathcal{B B}_{+}(n)$, to be the polytope

$$
\mathcal{B B}_{+}(n):=\mathcal{B B}(n) \cap \mathbb{R}_{+}^{n \times n}
$$

A simple way to view this as an anti-blocking polytope is via matching polytopes. Given a graph $G=([d], E)$, a matching is a set $M \subseteq E$ such that $e \cap e^{\prime}=\varnothing$ for any 
two distinct $e, e^{\prime} \in M$. The corresponding matching polytope is

$$
\operatorname{Mat}(G):=\operatorname{conv}\left\{\mathbf{1}_{M}: M \subseteq E \text { a matching }\right\} \subset \mathbb{R}^{E}
$$

If $G$ is a bipartite graph, then the matching polytope is easy to describe. For $v \in[d]$ let $\delta(v) \subseteq E$ denote the edges incident to $v$.

Theorem 5.4 ([64, Sec. 8.11]) For bipartite graphs $G$ the matching polytope is given by

$$
\operatorname{Mat}(G)=\left\{\boldsymbol{x} \in \mathbb{R}_{+}^{E}: \boldsymbol{x}(\delta(v)) \leq 1 \text { for all } v \in[d]\right\}
$$

As a simple consequence, we get

Corollary $5.5 \mathcal{B B}_{+}(n)$ is the matching polytope of the complete bipartite graph $K_{n, n}$ on $2 n$ vertices.

Proof We can identify the edges of $K_{n, n}$ with $[n] \times[n]$. Every matrix $M \in \mathcal{B B}_{+}(n) \cap$ $\{0,1\}^{n \times n}$ is a partial permutation matrix and therefore contains at most one 1 in every row and column. It follows that the set $\left\{(i, j): M_{i j}=1\right\}$ is a matching of $K_{n, n}$ and every matching arises that way. Since $\mathcal{B B}_{+}(n)$ and $\operatorname{Mat}\left(K_{n, n}\right)$ are both $0 / 1$-polytopes, this proves the claim.

It follows from the description given in Theorem 5.4 and the definition of compressed polytopes that matching polytopes of bipartite graphs are compressed. Hence, by Proposition 4.4, Mat $(G)$ is the stable set polytope of a perfect graph. The graph in question is the line graph $L(G)$ on the vertex set $E$ and edge $e e^{\prime}$ whenever $e \cap e^{\prime} \neq \varnothing$. It is clear that $M$ is a matching in $G$ if and only if $M$ is a stable set in $L(G)$. If $L(G)$ is perfect, then $G$ is called a line perfect graph. From Lovász' Theorem 4.1 one can then infer $\operatorname{Mat}(G)=P_{L(G)}$ and hence bipartite graphs are line perfect; cf. [49, Thm. 2].

The polytope $\mathcal{B B}_{+}(n)$ is the stable set polytope of $L\left(K_{n, n}\right)=K_{n} \square K_{n}$, the Cartesian product of complete graphs, which is the graph of legal moves of a rook on an $n$-by- $n$ chessboard and thus called a rook graph. Since all vertices in $K_{n, n}$ have the same degree, it follows that all maximal cliques in $K_{n} \square K_{n}$ have size $n$ and from Proposition 4.5 we conclude the following.

Corollary 5.6 The polytope $\mathcal{B B}_{+}(n)$ is Gorenstein.

For two matrices $A, B \in \mathbb{R}^{d \times d}$ we denote by $\langle A, B\rangle=\operatorname{tr}\left(A^{t} B\right)$ the Frobenius inner product. Also, for vectors $\boldsymbol{u}, \boldsymbol{v} \in \mathbb{R}^{d}$ let us write $\boldsymbol{u} \otimes \boldsymbol{v} \in \mathbb{R}^{d \times d}$ for the matrix with $(\boldsymbol{u} \otimes \boldsymbol{v})_{i j}=u_{i} v_{j}$.

Corollary 5.7 The polytope $\mathcal{B B}(n)$ is an unconditional reflexive polytope. Its facetdefining inequalities are given by

$$
\left\langle A, \sigma \otimes \boldsymbol{e}_{i}\right\rangle \leq 1 \quad \text { and } \quad\left\langle A, \boldsymbol{e}_{i} \otimes \sigma\right\rangle \leq 1
$$

for all $i=1, \ldots, n$ and $\sigma \in\{ \pm 1\}^{n}$. 
The inequality description of this polytope was previously obtained in [50] using the notion of Birkhoff tensors.

Proof We deduce that $\mathcal{B B}(n)$ is reflexive by appealing to Theorem 4.6, using the fact that $\mathcal{B B}(n)$ is unconditional and $\mathcal{B B}{ }_{+}(n)=\mathcal{B B}(n) \cap \mathbb{R}_{+}^{n \times n}$ is the stable set polytope of a perfect graph as discussed above. We obtain the inequality description by applying Proposition 3.1 and Theorem 5.4.

The dual $\mathcal{C}(n):=\mathcal{B B}(n)^{*}$ is the unconditional reflexive polytope associated with the graph $\overline{K_{n} \square K_{n}}$. The corresponding anti-blocking polytope $\mathcal{C}_{+}(n)=P_{\overline{K_{n} \square K_{n}}}$ also has the nice property that all cliques have the same size $n$ and hence Proposition 4.5 applies.

Corollary 5.8 The polytope $\mathcal{C}_{+}(n)$ is Gorenstein.

By Theorems 3.4 and 4.6, and Proposition 4.4, we have the following unimodality results.

Corollary 5.9 For any $n \in \mathbb{Z}_{\geq 1}$, we have that $h^{*}(\mathcal{B B}(n)), h^{*}\left(\mathcal{B B}_{+}(n)\right), h^{*}(\mathcal{C}(n))$, and $h^{*}\left(\mathcal{C}_{+}(n)\right)$ are unimodal.

Let us conclude this section with some enumerative data. The polytope $\mathcal{B B}(n)$ has $2^{n} n$ ! vertices and $n 2^{n+1}$ facets. In contrast, the vertices of $\mathcal{B} \mathcal{B}_{+}(n)$ are in bijection to partial permutations of $[n]$. Hence $\mathcal{B B}_{+}(n)$ has $n ! \sum_{i=0}^{n} 1 / i$ ! many vertices but only $n^{2}+2 n$ facets. The polytope $\mathcal{C}_{+}(n)$ has $n 2^{n+1}-(n+1)^{2}$ many vertices and $n^{2}+n$ ! facets. We used NORMALIZ [18] to compute the normalized volume and $h^{*}$-vectors of these polytopes; see Tables 2, 3, 4, and 5. Given the dimension and volumes of these polytopes, our computational resources were quite quickly exhausted. Note that $\mathcal{B B}(3)$ and $\mathcal{C}(3)$ have precisely the same Ehrhart data and normalized volume and in fact it is straightforward to verify that $\mathcal{B B}(3)$ and $\mathcal{C}(3)$ are unimodularly equivalent.

Using Theorem 3.6 and Corollary 3.7, we get a lower bound on the volume of $\mathcal{B B}_{+}(5)$ and $\mathcal{B B}(5)$, respectively. We get that

$$
\begin{aligned}
\operatorname{Vol}\left(\mathcal{B B}_{+}(5)\right) & >30,637,007,047,800 \\
\operatorname{Vol}(\mathcal{B B}(5)) & >1,028,007,369,668,940,603,880
\end{aligned}
$$

are bounds on the number of simplices in a unimodular triangulation.

\section{CIS Graphs and Compressed Gale-dual Pairs of Polytopes}

The notion of Gale-dual pairs was introduced in [32]. Given two polytopes $P, Q \subset \mathbb{R}^{d}$, we say that these polytopes form a Gale-dual pair if 
Table $2 \mathcal{B B}_{+}(n)$

\begin{tabular}{lll}
\hline$n$ & $\operatorname{Vol}\left(\mathcal{B B}_{+}(n)\right)$ & $h^{*}(\mathcal{B B}+(n))$ \\
\hline 1 & 1 & 1 \\
2 & 4 & $(1,2,1)$ \\
3 & 642 & $(1,24,156,280,156,24,1)$ \\
4 & 12065248 & $(1,192,9534,151856,975793,2860752,4069012$, \\
& & $2860752,975793,151856,9534,192,1)$ \\
\hline
\end{tabular}

Table $3 \mathcal{B B}(n)$

\begin{tabular}{lll}
\hline$n$ & $\operatorname{Vol}(\mathcal{B B}(n))$ & $h^{*}(\mathcal{B B}(n))$ \\
\hline 1 & 2 & $(1,1)$ \\
2 & 64 & $(1,12,38,12,1)$ \\
3 & 328704 & $(1,129,4482,40844,118950,118950,40844,4482,129,1)$ \\
4 & 790708092928 & $?$ \\
\hline
\end{tabular}

Table $4 \mathcal{C}_{+}(n)$

\begin{tabular}{lll}
\hline$n$ & $\operatorname{Vol}\left(\mathcal{C}_{+}(n)\right)$ & $h^{*}\left(\mathcal{C}_{+}(n)\right)$ \\
\hline 1 & 1 & 1 \\
2 & 6 & $(1,4,1)$ \\
3 & 642 & $(1,24,156,280,156,24,1)$ \\
4 & 2389248 & $(1,88,2656,34568,201215,562112,787968$ \\
5 & & $562112,201215,34568,2656,88,1)$ \\
\end{tabular}

Table $5 \mathcal{C}(n)$

\begin{tabular}{lll}
\hline$n$ & Vol $(\mathcal{C}(n))$ & $h^{*}(\mathcal{C}(n))$ \\
\hline 1 & 2 & $(1,1)$ \\
2 & 96 & $(1,20,54,20,1)$ \\
3 & 328704 & $(1,129,4428,40844,118950,118950,40844,4428,129,1)$ \\
4 & 156581756928 & $(1,592,110136,8093168,222332060,2558902352$, \\
& & $13699272072,36553260912,50497814342,36553260912$, \\
& & $136992720722558902352,222332060,8093168,110136,592,1)$ \\
5 & 16988273098107125760 & $?$ \\
\hline
\end{tabular}




$$
\begin{aligned}
& P=\left\{\boldsymbol{x} \in \mathbb{R}_{+}^{d}:\langle\boldsymbol{x}, \boldsymbol{y}\rangle=1 \text { for } \boldsymbol{y} \in V(Q)\right\} \text { and } \\
& Q=\left\{\boldsymbol{x} \in \mathbb{R}_{+}^{d}:\langle\boldsymbol{x}, \boldsymbol{y}\rangle=1 \text { for } \boldsymbol{y} \in V(P)\right\} .
\end{aligned}
$$

Primary examples of Gale-dual pairs of polytopes are the Birkhoff polytope $\mathcal{B}_{n}$, the convex hull of permutation matrices $M_{\tau}$, and the Gardner polytope $\mathcal{G}_{n}$, which is the polytope of all nonnegative matrices $A \in \mathbb{R}_{+}^{n \times n}$ such that $\left\langle M_{\tau}, A\right\rangle=1$ for all permutation matrices $M_{\tau}$. Both polytopes are compressed, Gorenstein lattice polytopes of codegree $n$. The question raised in [32] was if there were other Gale-dual pairs with (a subset of) these properties. In this section we briefly outline a construction for compressed Gale-dual pairs of polytopes.

Following [2], we call $G=([d], E)$ a $C I S$ graph if $C \cap S \neq \varnothing$ for every inclusionmaximal clique $C$ and inclusion-maximal stable set $S$. For brevity, we refer to those as maximal cliques and stable sets, respectively. For example, if $B$ is a bipartite graph with perfect matching, i.e., a matching covering all vertices, then the line graph $L(G)$ is CIS. Another class of examples is given by a theorem of Grillet [35]. Let $\Pi=([d], \preceq)$ be a partially ordered set. The comparability graph of $\Pi$ is the simple graph $G_{\prec}=([d], E)$ with $i j \in E$ if $i \prec j$ or $j \prec i$. Comparability graphs are known to be perfect [44]. The bull graph is the graph with vertices $a, b, c, d, e$ and edges $a b, b c, c d, d e, b d$.

Theorem 6.1 ([35]) Let $(\Pi, \preceq)$ be a poset with comparability graph $G$. Then $G$ is CIS if every induced 4-path is contained in an induced bull graph.

The wording in graph-theoretic terms is due to Berge; see [74] for extensions.

Proposition 6.2 Let $G$ be a perfect CIS graph. Then

$$
\begin{aligned}
& P=\operatorname{conv}\left(\boldsymbol{1}_{S}: S \text { a maximal stable set of } G\right) \\
& Q=\operatorname{conv}\left(\boldsymbol{1}_{C}: C \text { a maximal clique of } G\right)
\end{aligned}
$$

is a Gale-dual pair of compressed polytopes.

Proof For a stable set $S$ and a clique $C$, we have that $S \cap C \neq \varnothing$ if and only if $\left\langle\mathbf{1}_{C}, \mathbf{1}_{S}\right\rangle=1$. Let $P_{G}$ be the stable set polytope of $G$. It follows from Theorem 4.1 that the vertices of

$$
P^{\prime}:=P_{G} \cap \bigcap_{C \text { maximal clique }}\left\{\boldsymbol{x} \in \mathbb{R}_{+}^{n}: \boldsymbol{x}(C)=\left\langle\mathbf{1}_{C}, \boldsymbol{x}\right\rangle=1\right\}
$$

are of the form $\mathbf{1}_{S}$ for stable sets meeting every maximal clique non-trivially. Note that a stable set $S$ of the CIS graph $G$ is maximal if $S \cap C \neq \varnothing$ for every maximal clique $C$. Hence $P=P^{\prime}$ and $P$ is a face of $P_{G}$. Since faces of compressed polytopes are compressed, it follows that $P$ is compressed. The complement graph $\bar{G}$ is also a perfect CIS graph and the same argument applied to $Q$ completes the proof.

Note that both examples above are perfect CIS graphs. This shows that compressed (lattice) Gale-dual pairs are not rare. Recall that a graph $G$ is well-covered if every maximal stable set has the same size and $G$ is co-well-covered if $\bar{G}$ is well-covered. 
Theorem 6.1 and its generalization in [74] allow for the construction of perfect CIS graphs which are well-covered and co-well-covered (for example, by taking ordinal sums of antichains). Moreover, the recent paper [28] gives classes of examples of wellcovered and co-well-covered CIS graphs. This is a potential source of compressed Gorenstein Gale-dual pairs but we were unable to identify the perfect graphs in these families.

Theorem 4.6 implies that if $(F, G)$ is a Gale-dual pair of Proposition 6.2, then there is a (unconditional) reflexive polytope such that $F \subset P$ and $G \subset P^{*}$ are dual faces.

Question 6.3 Is it true that every Gale-dual pair $(F, G)$ appears as dual faces of some reflexive polytope $P$ ?

\section{Chain Polytopes and Gröbner Bases}

Given a lattice polytope $P \subset \mathbb{R}^{d}$, the existence of regular triangulations, particularly those which are unimodular and flag, has direct applications to the associated toric ideal of $P$. In this section, we will discuss how certain Gröbner bases of the toric ideal of an anti-blocking polytope can be extended to Gröbner bases of the associated unconditional polytope. In particular, we provide an explicit description of Gröbner bases for unconditional polytopes arising from the special class of anti-blocking polytopes called chain polytopes. We refer the reader to the wonderful books [23] and [71] for background on Gröbner bases and toric ideals.

Let $Z:=P \cap \mathbb{Z}^{d}$. The toric ideal associated to $P$ is the ideal $I_{P} \subset \mathbb{C}\left[x_{p}: p \in Z\right]$ with generators

$$
x_{\boldsymbol{r}_{1}} x_{\boldsymbol{r}_{2}} \cdots x_{\boldsymbol{r}_{k}}-x_{\boldsymbol{s}_{1}} x_{\boldsymbol{s}_{2}} \cdots x_{\boldsymbol{s}_{k}}
$$

where $\boldsymbol{r}_{1}, \ldots, \boldsymbol{r}_{k}, \boldsymbol{s}_{1}, \ldots, \boldsymbol{s}_{k} \in Z$ are lattice points such that $\boldsymbol{r}_{1}+\cdots+\boldsymbol{r}_{k}=\boldsymbol{s}_{1}+$ $\cdots+s_{k}$. If we denote the two multisets of points by $R$ and $S$, we simply write $x^{R}-x^{S}$. A celebrated result of Sturmfels [71, Thm. 8.3] states that the regular triangulations $\mathcal{T}$ of $P$ (with vertices in $Z$ ) are in correspondence with (reduced) Gröbner bases of $I_{P}$. The heights inducing the triangulation yield a term order on $\mathbb{C}\left[x_{\boldsymbol{p}}: \boldsymbol{p} \in Z\right]$ and we write $x^{R}-x^{S}$ to emphasize that $x^{R}$ is the leading term. We set the following result on record, which reflects the content of Theorem 8.3 and Corollary 8.9 of [71]. For details on this algebraic-geometric correspondence outlined above, we recommend the very accessible Chapter 8 of Sturmfels' book [71].

Theorem 7.1 Let $P \subset \mathbb{R}^{d}$ be a lattice polytope and let $\mathcal{T}$ be a regular and unimodular triangulation. Then a reduced Gröbner basis of $I_{P}$ is given by the collection of monomials

$$
\underline{x^{R}}-x^{S},
$$

where $R \subset P \cap \mathbb{Z}^{d}$ is a minimal non-face, $S$ is a multisubset of $P \cap \mathbb{Z}^{d}$ such that $\sum R=\sum S$ and $\operatorname{conv}(S)$ is a face of some simplex in $\mathcal{T}$. In particular, $\mathcal{T}$ is flag if and only if the leading terms are quadratic and square-free. 
Let $\mathcal{T}$ be a unimodular triangulation of $P$. Given any lattice point $\boldsymbol{p} \in 2 P$ there are unique $\boldsymbol{p}^{(1)}, \boldsymbol{p}^{(2)} \in Z$ such that $\boldsymbol{p}=\boldsymbol{p}^{(1)}+\boldsymbol{p}^{(2)}$ and conv $\left(\boldsymbol{p}^{(1)}, \boldsymbol{p}^{(2)}\right)$ is a face of a simplex in $\mathcal{T}$. Note that $\boldsymbol{p}^{(1)}$ and $\boldsymbol{p}^{(2)}$ do not have to be distinct points. Let us call two points $\boldsymbol{p}, \boldsymbol{q} \in \mathbb{R}^{d}$ separable if $p_{i}$ and $q_{i}$ have different signs for some $i=1, \ldots, d$. Together with Theorem 3.2, this yields the following description of a Gröbner basis for unconditional reflexive polytopes.

Theorem 7.2 Let $P \subset \mathbb{R}^{d}$ be an anti-blocking polytope with a regular and unimodular triangulation and let $\underline{x^{R_{i}}}-x^{S_{i}}$ for $i=1, \ldots, m$ be the associated Gröbner basis for $I_{P}$. A Gröbner basis associated to the toric ideal of UP is given by the binomials

$$
\underline{x^{\sigma R_{i}}}-x^{\sigma S_{i}}
$$

for $i=1, \ldots, m$ and $\sigma \in\{ \pm 1\}^{d}$, and

$$
\underline{x^{p} x^{q}}-x^{\sigma e^{(1)}} x^{\sigma e^{(2)}}
$$

for any separable $\boldsymbol{p}, \boldsymbol{q} \in U P \cap \mathbb{Z}^{d}$ and $\sigma \in\{ \pm 1\}^{d}$ such that $\sigma(\boldsymbol{p}+\boldsymbol{q})=\boldsymbol{e} \in 2 P$.

Proof Theorem 3.2 states that the regular and unimodular triangulation $\mathcal{T}$ of $P$ induces a regular and unimodular triangulation $U \mathcal{T}$ of $U P$. It follows from Theorem 3.2 (iii) that a minimal non-face $R$ of $\cup \mathcal{T}$ is of the form $R=\sigma R^{\prime}$, where $R^{\prime}$ is a non-face of $\mathcal{T}$ and $\sigma \in\{ \pm 1\}^{d}$, or it is of the form $R=\{\boldsymbol{p}, \boldsymbol{q}\}$ for separable $\boldsymbol{p}, \boldsymbol{q} \in Z:=\cup P \cap \mathbb{Z}^{d}$.

In order to apply Theorem 7.1, we need to determine for every minimal non-face $R$ the multisubset $S$ of $Z$ such that $x^{R}-x^{S} \in I_{\cup P}$. If $R=\sigma R_{i}$ for some minimal non-face $R_{i}$, then we can take $S=\sigma S_{i}$. If $R=\{\boldsymbol{p}, \boldsymbol{q}\}$, then there is some $\sigma \in\{ \pm 1\}^{d}$, such that $\sigma(\boldsymbol{p}+\boldsymbol{q})=\boldsymbol{e} \in 2 P$. It follows that $\boldsymbol{p}+\boldsymbol{q} \in \operatorname{conv}\left(\sigma \boldsymbol{e}^{(1)}, \sigma \boldsymbol{e}^{(2)}\right)$, which is a face of some simplex of $U \mathcal{T}$. Hence we can take $S=\left\{\sigma \boldsymbol{e}^{(1)}, \sigma \boldsymbol{e}^{(2)}\right\}$.

A prominent class of perfect graphs $G$ for which regular, unimodular triangulations of $P_{G}$, as well as Gröbner bases for $I_{P_{G}}$, are well understood are comparability graphs of finite posets. Let $\Pi=([d], \preceq)$ be a partially ordered set with comparability graph $G_{\prec}$. The stable set polytopes of comparability graphs were studied by Stanley [68] under the name chain polytopes and are denoted by $\mathcal{C}(\Pi)$. An antichain in $\Pi$ is a collection of pairwise uncomparable elements. The vertices of $P_{G_{\prec}}$ are precisely the points $\mathbf{1}_{A}$, where $A$ is an antichain. Let $\mathcal{A}(\Pi)$ denote the collection of antichains. A pulling triangulation of $P_{G_{\prec}}$ can be explicitly described (see Sect. 4.1 in [21] for exposition and details). The corresponding (reverse lexicographic) Gröbner basis was described by Hibi [40]. Following [21], we define

$$
A \sqcup A^{\prime}:=\min \left(A \cup A^{\prime}\right) \text { and } A \sqcap A^{\prime}:=\left(A \cap A^{\prime}\right) \cup\left(\max \left(A \cup A^{\prime}\right) \backslash \min \left(A \cup A^{\prime}\right)\right) \text {, }
$$

where min and max are taken with respect to the partial order $\preceq$. We call two antichains $A, A^{\prime}$ uncomparable if $\max \left(A \cup A^{\prime}\right)$ is not a subset of $A$ and not of $A^{\prime}$. To ease notation, we identify variables $x_{A}$ in $\mathbb{C}\left[x_{A}: A \in \mathcal{A}(\Pi)\right]$ with symbols $[A]$. 
Theorem 7.3 ([40,68]) Let $\Pi$ be a poset and $\mathcal{C}(\Pi)$ its chain polytope. A Gröbner basis for $I_{\mathcal{C}(\Pi)}$ is given by the binomials

$$
\underline{[B] \cdot\left[B^{\prime}\right]}-\left[B \sqcup B^{\prime}\right] \cdot\left[B \sqcap B^{\prime}\right],
$$

for all uncomparable antichains $B, B^{\prime} \in \mathcal{A}(\Pi)$. The corresponding triangulation of $\mathcal{C}(\Pi)$ is regular, unimodular, and flag.

We define the unconditional chain polytope $\cup \mathcal{C}(\Pi)$ as the unconditional reflexive polytope associated to $G_{\prec}$. These polytopes were independently introduced by Ohsugi and Tsuchiya [58] under the name enriched chain polytopes. The lattice points in $\cup \mathcal{C}(\Pi)$ are uniquely given by

$$
\mathbf{1}_{B}-2 \cdot \mathbf{1}_{A},
$$

where $A \subseteq B$ are antichains. We write $B-A$ for the pair $A \subseteq B$ of antichains. In the following, we slightly abuse notation and write $B-C$ instead of $B-(B \cap C)$ for antichains $B, C$. We get a description of the vertices of $\cup \mathcal{C}(\Pi)$ from Proposition 3.1: Every vertex of $\cup \mathcal{C}(\Pi)$ is of the form $\boldsymbol{v}=\sigma \mathbf{1}_{B}$ for some inclusion-maximal antichain $B \subseteq \Pi$. Setting $A:=\left\{i \in B: v_{i}<0\right\}$, we deduce that the vertices of $\cup \mathcal{C}(\Pi)$ are uniquely given by $B-A$ where $B$ is an inclusion-maximal antichain.

We call $B-A$ and $B^{\prime}-A^{\prime}$ separable if the corresponding points are separable. We also extend the two operations introduced above:

$$
\begin{aligned}
& (B-A) \sqcup\left(B^{\prime}-A^{\prime}\right):=\left(B \sqcup B^{\prime}\right)-\left(A \sqcup A^{\prime}\right), \\
& (B-A) \sqcap\left(B^{\prime}-A^{\prime}\right):=\left(B \sqcap B^{\prime}\right)-\left(A \sqcap A^{\prime}\right) .
\end{aligned}
$$

The following result was also obtained in [58].

Theorem 7.4 Let $\Pi=([d], \preceq)$ be a finite poset and $I_{\cup \mathcal{C}(\Pi)}$ the toric ideal associated to the unconditional chain polytope $U \mathcal{C}(\Pi)$. Then a reduced Gröbner basis is given by the binomials

$$
\underline{[B-E] \cdot\left[B^{\prime}-E\right]}-\left[\left(B \sqcup B^{\prime}\right)-E\right] \cdot\left[\left(B \sqcap B^{\prime}\right)-E\right],
$$

for all uncomparable $B, B^{\prime} \in \mathcal{A}(\Pi)$ and $E \subseteq B \cup B^{\prime}$ as well as

$$
\underline{[B-A] \cdot\left[B^{\prime}-A^{\prime}\right]}-\left[(C-D) \sqcup\left(C^{\prime}-D^{\prime}\right)\right] \cdot\left[(C-D) \sqcap\left(C^{\prime}-D^{\prime}\right)\right],
$$

where $B-A$ and $B^{\prime}-A^{\prime}$ are separable and

$$
\begin{array}{rlrl}
C & :=\left(B \backslash B^{\prime}\right) \cup\left(A \cap A^{\prime}\right), & D & :=\left(A \backslash B^{\prime}\right) \cup\left(A \cap A^{\prime}\right), \\
C^{\prime} & :=\left(B^{\prime} \backslash B\right) \cup\left(A \cap A^{\prime}\right), & D^{\prime}:=\left(A^{\prime} \backslash B\right) \cup\left(A \cap A^{\prime}\right) .
\end{array}
$$

Proof We apply Theorem 7.2 using the Gröbner basis of the toric ideal of $\mathcal{C}(\Pi)$ provided by Theorem 7.3. This provides the first set of binomials and we only have 
to argue on the binomials with leading terms coming from separable pairs $B-A$ and $B^{\prime}-A^{\prime}$.

The Gröbner basis given in Theorem 7.3 tells us how to find the decomposition $\boldsymbol{e}^{(1)}+\boldsymbol{e}^{(2)}$ of a point $\boldsymbol{e} \in 2 \mathcal{C}(\Pi)$. Indeed, the minimal non-faces of the triangulation are given by the leading terms and correspond to uncomparable pairs of antichains $E, E^{\prime} \in \mathcal{A}(\Pi)$. The point $\mathbf{1}_{E}+\mathbf{1}_{E^{\prime}}$ can then be written as $\mathbf{1}_{E \sqcup E^{\prime}}+\mathbf{1}_{E \sqcap E^{\prime}}$. The antichains $E \sqcup E^{\prime}, E \sqcap E^{\prime}$ are comparable and thus conv $\left(\mathbf{1}_{E \sqcup E^{\prime}}, \mathbf{1}_{E \sqcap E^{\prime}}\right)$ is a face of a simplex in the triangulation.

By our discussion above, every point in $U \mathcal{C}(\Pi)$ is of the form $\mathbf{1}_{B}-2 \cdot \mathbf{1}_{A}$ for $B \in \mathcal{A}(\Pi)$ and $A \subseteq B$. Now, for $B-A$ and $B^{\prime}-A^{\prime}$ separable, we have

$$
\boldsymbol{p}=\left(\mathbf{1}_{B}-2 \cdot \mathbf{1}_{A}\right)+\left(\mathbf{1}_{B^{\prime}}-2 \cdot \mathbf{1}_{A^{\prime}}\right)=\left(\mathbf{1}_{C}-2 \cdot \mathbf{1}_{D}\right)+\left(\mathbf{1}_{C^{\prime}}-2 \cdot \mathbf{1}_{D^{\prime}}\right) .
$$

Note that $C-D$ and $C^{\prime}-D^{\prime}$ are not separable and the pairs $(C-D) \sqcup\left(C^{\prime}-D^{\prime}\right)$ and $(C-D) \sqcap\left(C^{\prime}-D^{\prime}\right)$ give a suitable representation of $\boldsymbol{p}$ with respect to the triangulation of $\cup \mathcal{C}(\Pi)$.

\section{Concluding Remarks}

\subsection{A Blaschke-Santaló Inequality}

The Blaschke-Santaló inequality [63, Sect. 10.7] implies that for a centrally-symmetric convex body $K \subset \mathbb{R}^{d}$,

$$
\operatorname{vol}(K) \cdot \operatorname{vol}\left(K^{*}\right) \leq \operatorname{vol}\left(B_{d}\right)^{d}
$$

where $B_{d}$ is the Euclidean unit ball. Equality is attained precisely when $K$ is an ellipsoid. Based on computations for up to 9 vertices, we conjecture the following.

Conjecture 8.1 For every $n \geq 1$, there is a unique perfect graph $G$ on $n$ vertices such that $\operatorname{vol}\left(U P_{G}\right) \cdot \operatorname{vol}\left(U P_{\bar{G}}\right)$ is maximal.

For $n=3,4,5$, the unique maximizer is the path on $n$ vertices. For $n=6$ the unique maximizer is a 6 -cycle and for $n=7$ the maximizer is obtained by adding a dangling edge to the 6-cycle. For $n=8$ the graph in question is a 6-cycle with an additional 4-path connecting two antipodal vertices but for $n=9$ the graph is much more complicated.

\subsection{Birkhoff Polytopes of Other Types}

It is natural to look at Birkhoff-type polytopes of other finite irreducible Coxeter groups. Since the type- $B$ and the type- $C$ Coxeter groups are equal, we get the same polytope. Recall that the type- $D$ Coxeter group $D_{n}$ is the subgroup of $B_{n}$ with permutations with an even number of negative entries. We can construct the type-D Birkhoff polytope, $\mathcal{B D}(n)$, to be the convex hull of signed permutation matrices with an even 


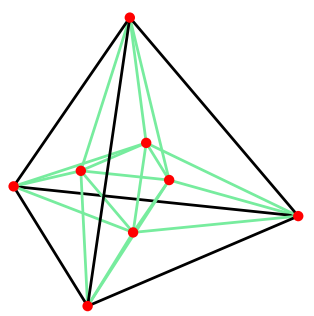

Fig. 2 Schlegel diagram for $\mathcal{B B}(2)$

number of negative entries. As one may suspect from this construction, the omission of all lattice points in various orthants which occurs in $\mathcal{B D}(n)$ ensures that it cannot be an OLP polytope and is thus not subject to any of our general theorems. When $n=2$ and $n=3, \mathcal{B D}(n)$ is a reflexive polytope, but $\mathcal{B D}(3)$ does not have the IDP. Moreover, $\mathcal{B D}(4)$ fails to be reflexive.

Additionally, one could consider Birkhoff constructions for Coxeter groups of exceptional type, in particular $E_{6}, E_{7}$ and $E_{8}$ (see, e.g., [12]). While we did not consider these polytopes in our investigation, we do raise the following question:

Question 8.2 Do the Birkhoff polytope constructions for $E_{6}, E_{7}$, and $E_{8}$ have the IDP? Are these polytopes reflexive? Do they have other interesting properties?

\subsection{Future Directions}

In addition to considering Birkhoff polytopes of other types and connections to Gale duality as discussed above, there are several immediate avenues for further research. Coxeter groups are of great interest in the broader community of algebraic and geometric combinatorics (see, e.g., [12]) and it would be interesting if Coxeter-theoretic insights can be gained from the geometry of $\mathcal{B B}(n)$.

An additional future direction is to consider applications of the orthant-lattice property, particularly those of Theorem 3.2 and Remark 3.5. One potentially fruitful avenue is an application to reflexive smooth polytopes. Recall that a lattice polytope $P \subset \mathbb{R}^{d}$ is simple if every vertex of $P$ is contained in exactly $d$ edges (see, e.g., [75]). A simple polytope $P$ is called smooth if the primitive edge directions generate $\mathbb{Z}^{d}$ at every vertex of $P$. Smooth polytopes are particularly of interest due to a conjecture commonly attributed to Oda [53]:

Conjecture 8.3 (Oda) If $P$ is a smooth polytope, then $P$ has the IDP.

This conjecture is not only of interest in the context of Ehrhart theory, but also in toric geometry. One potential strategy is to consider similar constructions to OLP polytopes for smooth reflexive polytopes to make progress towards this problem. As a first step, we pose the following question:

Question 8.4 Are all smooth reflexive polytopes OLP polytopes?

Furthermore, regarding reflexive OLP polytopes one can ask the question: 
Question 8.5 Given a reflexive OLP polytope $P$, under what conditions can we guarantee that $P^{*}$ is a reflexive OLP polytope?

By (2), this has a positive answer when $P$ is an unconditional reflexive polytope. However, there are multiple examples of failure in general even in dimension 2 (see Fig. 1).

Acknowledgements Open Access funding provided by Projekt DEAL. The first two authors would like to thank Matthias Beck, Benjamin Braun, and Jan Hofmann for helpful comments and suggestions for this work. Furthermore, Fig. 2 was created by Benjamin Schröter. Additionally, the authors thank Takayuki Hibi and Akiyoshi Tsuchiya for organizing the 2018 Summer Workshop on Lattice Polytopes at Osaka University where this work began. The third author thanks Kolja Knauer and Sebastian Manecke for insightful conversations.

Open Access This article is licensed under a Creative Commons Attribution 4.0 International License, which permits use, sharing, adaptation, distribution and reproduction in any medium or format, as long as you give appropriate credit to the original author(s) and the source, provide a link to the Creative Commons licence, and indicate if changes were made. The images or other third party material in this article are included in the article's Creative Commons licence, unless indicated otherwise in a credit line to the material. If material is not included in the article's Creative Commons licence and your intended use is not permitted by statutory regulation or exceeds the permitted use, you will need to obtain permission directly from the copyright holder. To view a copy of this licence, visit http://creativecommons.org/licenses/by/4.0/.

\section{References}

1. Adiprasito, K., Huh, J., Katz, E.: Hodge theory for combinatorial geometries. Ann. Math. 188(2), 381-452 (2018)

2. Andrade, D.V., Boros, E., Gurvich, V.: On graphs whose maximal cliques and stable sets intersect. In: Optimization Problems in Graph Theory. Springer Optim. Appl., vol. 139, pp. 3-63. Springer, Cham (2018)

3. Artstein-Avidan, S., Sadovsky, S., Sanyal, R.: Volume and mixed volume inequalities for locally antiblocking bodies (2020, in preparation)

4. Athanasiadis, Ch.A.: Ehrhart polynomials, simplicial polytopes, magic squares and a conjecture of Stanley. J. Reine Angew. Math. 583, 163-174 (2005)

5. Bapat, R.B., Raghavan, T.E.S.: Nonnegative Matrices and Applications. Encyclopedia of Mathematics and its Applications, vol. 64. Cambridge University Press, Cambridge (1997)

6. Batyrev, V.V.: Dual polyhedra and mirror symmetry for Calabi-Yau hypersurfaces in toric varieties. J. Algebr. Geom. 3(3), 493-535 (1994)

7. Beck, M., Haase, Ch., Sam, S.V.: Grid graphs, Gorenstein polytopes, and domino stackings. Graphs Comb. 25(4), 409-426 (2009)

8. Beck, M., Pixton, D.: The Ehrhart polynomial of the Birkhoff polytope. Discrete Comput. Geom. 30(4), 623-637 (2003)

9. Beck, M., Robins, S.: Computing the Continuous Discretely. Undergraduate Texts in Mathematics. Springer, New York (2015)

10. Beck, M., Sanyal, R.: Combinatorial Reciprocity Theorems. Graduate Studies in Mathematics, vol. 195. American Mathematical Society, Providence (2018)

11. Birkhoff, G.: Three observations on linear algebra. Univ. Nac. Tucumán. Rev. A 5, 147-151 (1946). (in Spanish)

12. Björner, A., Brenti, F.: Combinatorics of Coxeter Groups. Graduate Texts in Mathematics, vol. 231. Springer, New York (2005)

13. Bollobás, B., Brightwell, G.R.: Convex bodies, graphs and partial orders. Proc. Lond. Math. Soc. 80(2), 415-450 (2000)

14. Brazitikos, S., Giannopoulos, A., Valettas, P., Vritsiou, B.-H.: Geometry of Isotropic Convex Bodies. Mathematical Surveys and Monographs, vol. 196. American Mathematical Society, Providence (2014) 
15. Brenti, F.: Log-concave and unimodal sequences in algebra, combinatorics, and geometry: an update. In: Jerusalem Combinatorics '93. Contemp. Math., vol. 178, pp. 71-89. American Mathematical Society, Providence (1994)

16. Bruns, W., Gubeladze, J.: Normality and covering properties of affine semigroups. J. Reine Angew. Math. 510, 161-178 (1999)

17. Bruns, W., Gubeladze, J.: Polytopes, Rings, and $K$-Theory. Springer Monographs in Mathematics. Springer, Dordrecht (2009)

18. Bruns, W., Ichim, B., Römer, T., Sieg, R., Söger, Ch.: Normaliz. Algorithms for rational cones and affine monoids. https://www.normaliz.uni-osnabrueck.de

19. Bruns, W., Römer, T.: $h$-vectors of Gorenstein polytopes. J. Comb. Theory Ser. A 114(1), 65-76 (2007)

20. Canfield, E.R., McKay, B.D.: The asymptotic volume of the Birkhoff polytope. Online J. Anal. Comb. 4 (2009)

21. Chappell, T., Friedl, T., Sanyal, R.: Two double poset polytopes. SIAM J. Discrete Math. 31(4), 23782413 (2017)

22. Cox, D.A.: Mirror symmetry and polar duality of polytopes. Symmetry 7(3), 1633-1645 (2015)

23. Cox, D.A., Little, J., O'Shea, D.: Ideals, Varieties, and Algorithms, Undergraduate Texts in Mathematics. Springer, Cham (2015)

24. Davis, R.: Ehrhart series of polytopes related to symmetric doubly-stochastic matrices. Electron. J. Comb. 22(2), 17 (2015)

25. De Loera, J.A., Liu, F., Yoshida, R.: A generating function for all semi-magic squares and the volume of the Birkhoff polytope. J. Algebr. Comb. 30(1), 113-139 (2009)

26. De Loera, J.A., Rambau, J., Santos, F.: Triangulations. Algorithms and Computation in Mathematics, vol. 25. Springer, Berlin (2010)

27. De Negri, E., Hibi, T.: Gorenstein algebras of Veronese type. J. Algebra 193(2), 629-639 (1997)

28. Dobson, E., Hujdurović, A., Milanič, M., Verret, G.: Vertex-transitive CIS graphs. Eur. J. Comb. 44(A), 87-98 (2015)

29. Ehrenborg, R., Hetyei, G., Readdy, M.: Simion's type $B$ associahedron is a pulling triangulation of the Legendre polytope. Discrete Comput. Geom. 60(1), 98-114 (2018)

30. Ehrhart, E.: Sur les polyèdres rationnels homothétiques a $n$ dimensions. C. R. Acad. Sci. Paris 254, 616-618 (1962)

31. Freij, R., Henze, M., Schmitt, M.W., Ziegler, G.M.: Face numbers of centrally symmetric polytopes produced from split graphs. Electron. J. Comb. 20(2), 32 (2013)

32. Fritsch, K., Heuer, J., Sanyal, R., Schulz, N.: The Martin Gardner polytopes. Am. Math. Mon. (accepted)

33. Fulkerson, D.R.: Blocking and anti-blocking pairs of polyhedra. Math. Program. 1, 168-194 (1971)

34. Fulkerson, D.R.: Anti-blocking polyhedra. J. Comb. Theory Ser. B 12, 50-71 (1972)

35. Grillet, P.A.: Maximal chains and antichains. Fund. Math. 65, 157-167 (1969)

36. Grötschel, M., Lovász, L., Schrijver, A.: Geometric Algorithms and Combinatorial Optimization. Algorithms and Combinatorics, vol. 2. Springer, Berlin (1993)

37. Haase, Ch., Paffenholz, A., Piechnik, L.C., Santos, F.: Existence of unimodular triangulations - positive results (2017). arXiv:1405.1687

38. Henk, M., Richter-Gebert, J., Ziegler, G.M.: Basic properties of convex polytopes. In: Goodman, J.E., O’Rourke, J., Tóth, C.D. (eds.): Handbook of Discrete and Computational Geometry, 3rd ed. CRC Press Ser. Discrete Math. Appl., pp. 383-413. CRC Press, Boca Raton (2017)

39. Hetyei, G.: Delannoy orthants of Legendre polytopes. Discrete Comput. Geom. 42(4), 705-721 (2009)

40. Hibi, T.: Distributive lattices, affine semigroup rings and algebras with straightening laws. In: Commutative Algebra and Combinatorics (Kyoto 1985). Adv. Stud. Pure Math., vol. 11, pp. 93-109. North-Holland, Amsterdam (1987)

41. Hibi, T.: Dual polytopes of rational convex polytopes. Combinatorica 12(2), 237-240 (1992)

42. Hibi, T., Matsuda, K., Tsuchiya, A.: Gorenstein Fano polytopes arising from order polytopes and chain polytopes (2015). arXiv:1507.03221

43. Hofmann, J.: Three Interesting Lattice Polytope Problems. PhD thesis, Freie Universität, Berlin (2018). https://refubium.fu-berlin.de/

44. Hougardy, S.: Classes of perfect graphs. Discrete Math. 306(19-20), 2529-2571 (2006)

45. Kreuzer, M., Skarke, H.: Classification of reflexive polyhedra in three dimensions. Adv. Theor. Math. Phys. 2(4), 853-871 (1998)

46. Kreuzer, M., Skarke, H.: Complete classification of reflexive polyhedra in four dimensions. Adv. Theor. Math. Phys. 4(6), 1209-1230 (2000) 
47. Lagarias, J.C., Ziegler, G.M.: Bounds for lattice polytopes containing a fixed number of interior points in a sublattice. Canad. J. Math. 43(5), 1022-1035 (1991)

48. Lovász, L.: Normal hypergraphs and the perfect graph conjecture. Discrete Math. 2(3), 253-267 (1972)

49. Maffray, F.: Kernels in perfect line-graphs. J. Comb. Theory Ser. B 55(1), 1-8 (1992)

50. McCarthy, N., Ogilvie, D., Spitkovsky, I., Zobin, N.: Birkhoff's theorem and convex hulls of Coxeter groups. Linear Algebra Appl. 347, 219-231 (2002)

51. Meyer, M.: Une caractérisation volumique de certains espaces normés de dimension finie. Israel J. Math. 55(3), 317-326 (1986)

52. von Neumann, J.: A certain zero-sum two-person game equivalent to the optimal assignment problem. In: Contributions to the Theory of Games, vol. 2. Annals of Mathematics Studies, vol. 28, pp. 5-12. Princeton University Press, Princeton (1953)

53. Oda, T.: Problems on Minkowski sums of convex lattice polytopes (2008). arXiv:0812.1418

54. Ohsugi, H.: Gorenstein cut polytopes. Eur. J. Comb. 38, 122-129 (2014)

55. Ohsugi, H., Hibi, T.: Convex polytopes all of whose reverse lexicographic initial ideals are squarefree. Proc. Am. Math. Soc. 129(9), 2541-2546 (2001)

56. Ohsugi, H., Hibi, T.: Special simplices and Gorenstein toric rings. J. Comb. Theory Ser. A 113(4), 718-725 (2006)

57. Ohsugi, H., Hibi, T.: Reverse lexicographic squarefree initial ideals and Gorenstein Fano polytopes. J. Commut. Algebra 10(2), 171-186 (2018)

58. Ohsugi, H., Tsuchiya, A.: Enriched chain polytopes (2019). arXiv:1812.02097

59. Paffenholz, A.: Faces of Birkhoff polytopes. Electron. J. Comb. 22(1), 1.67 (2015)

60. Reisner, S.: Minimal volume-product in Banach spaces with a 1-unconditional basis. J. Lond. Math. Soc. 36(1), 126-136 (1987)

61. Saint-Raymond, J.: Sur le volume des corps convexes symétriques. In: Initiation Seminar on Analysis: G. Choquet-M. Rogalski-J. Saint-Raymond, 20th Year: 1980/1981. Publ. Math. Univ. Pierre et Marie Curie, vol. 46, \# 11. Univ. Paris VI, Paris (1981)

62. Sanyal, R., Werner, A., Ziegler, G.M.: On Kalai's conjectures concerning centrally symmetric polytopes. Discrete Comput. Geom. 41(2), 183-198 (2009)

63. Schneider, R.: Convex Bodies: the Brunn-Minkowski Theory, 2nd ed. Encyclopedia of Mathematics and Its Applications, vol. 151. Cambridge University Press, Cambridge (2014)

64. Schrijver, A.: Theory of Linear and Integer Programming. Wiley-Interscience Series in Discrete Mathematics. Wiley, Chichester (1986)

65. Sloane, N.J.A.: The On-Line Encyclopedia of Integer Sequences (May 2019). https://oeis.org

66. Stanley, R.P.: Hilbert functions of graded algebras. Adv. Math. 28(1), 57-83 (1978)

67. Stanley, R.P.: Decompositions of rational convex polytopes. Ann. Discrete Math. 6, 333-342 (1980)

68. Stanley, R.P.: Two poset polytopes. Discrete Comput. Geom. 1(1), 9-23 (1986)

69. Stanley, R.P.: Log-concave and unimodal sequences in algebra, combinatorics, and geometry. In: Graph Theory and Its Applications: East and West (Jinan 1986). Ann. New York Acad. Sci., vol. 576, pp. 500-535. New York Acad. Sci., New York (1989)

70. Stanley, R.P.: A monotonicity property of $h$-vectors and $h^{*}$-vectors. Eur. J. Comb. 14(3), 251-258 (1993)

71. Sturmfels, B.: Gröbner Bases and Convex Polytopes. University Lecture Series, vol. 8. American Mathematical Society, Providence (1996)

72. Sullivant, S.: Compressed polytopes and statistical disclosure limitation. Tohoku Math. J. 58(3), 433445 (2006)

73. Tagami, M.: Gorenstein polytopes obtained from bipartite graphs. Electron. J. Comb. 17, 8 (2010)

74. Zang, W.: Generalizations of Grillet's theorem on maximal stable sets and maximal cliques in graphs. Discrete Math. 143(1-3), 259-268 (1995)

75. Ziegler, G.M.: Lectures on Polytopes. Graduate Texts in Mathematics, vol. 152. Springer, New York (1995)

Publisher's Note Springer Nature remains neutral with regard to jurisdictional claims in published maps and institutional affiliations. 